\title{
Cross-cultural music cognition: cognitive methodology applied to North Sami yoiks
}

\author{
Carol L. Krumhansl ${ }^{\mathrm{a}, \mathrm{b}, *}$, Pekka Toivanen ${ }^{\mathrm{a}}$, Tuomas Eerola ${ }^{\mathrm{a}}$, \\ Petri Toiviainen $^{\text {a }}$, Topi Järvinen ${ }^{a}$, Jukka Louhivuori ${ }^{\mathrm{a}}$ \\ ${ }^{\mathrm{a} U n i v e r s i t y}$ of Jyväskylä, Jyväskylä, Finland \\ ${ }^{\mathrm{b}}$ Department of Psychology, Cornell University, Ithaca, NY, USA
}

Received 16 September 1998; received in revised form 26 October 1999; accepted 15 February 2000

\begin{abstract}
This article is a study of melodic expectancy in North Sami yoiks, a style of music quite distinct from Western tonal music. Three different approaches were taken. The first approach was a statistical style analysis of tones in a representative corpus of 18 yoiks. The analysis determined the relative frequencies of tone onsets and two- and three-tone transitions. It also identified style characteristics, such as pentatonic orientation, the presence of two reference pitches, the frequency of large consonant intervals, and a relatively large set of possible melodic continuations. The second approach was a behavioral experiment in which listeners made judgments about melodic continuations. Three groups of listeners participated. One group was from the Sami culture, the second group consisted of Finnish music students who had learned some yoiks, and the third group consisted of Western musicians unfamiliar with yoiks. Expertise was associated with stronger veridical expectations (for the correct next tone) than schematic expectations (based on general style characteristics). Familiarity with the particular yoiks was found to compensate for lack of experience with the musical culture. The third approach simulated melodic expectancy with neural network models of the selforganizing map (SOM) type (Kohonen, T. (1997). Self-organizing maps (2nd ed.). Berlin: Springer). One model was trained on the excerpts of yoiks used in the behavioral experiment including the correct continuation tone, while another was trained with a set of Finnish folk songs and Lutheran hymns. The convergence of the three approaches showed that both listeners and the SOM model are influenced by the statistical distributions of tones and tone sequences. The listeners and SOM models also provided evidence supporting a core
\end{abstract}

\footnotetext{
* Corresponding author. Department of Psychology, Uris Hall, Cornell University, Ithaca, NY 14853, USA. Fax: +1-607-255-8433.

E-mail address: clk4@cornell.edu (C.L. Krumhansl).
}

0010-0277/00/\$ - see front matter (C) 2000 Elsevier Science B.V. All rights reserved. PII: S0010-0277(00)00068-8 
set of psychological principles underlying melody formation whose relative weights appear to differ across musical styles. (C) 2000 Elsevier Science B.V. All rights reserved.

Keywords: Music cognition; Expectation; Melody; Neural network model; Statistical learning

\section{Introduction}

Traditionally, cross-cultural studies of music have been the domain of ethnomusicology. Ethnomusicologists, often working in the anthropological tradition, have accepted certain ideas about music in ethnic cultures outside the Western tradition. These beliefs and hypotheses are most often based on field work lasting over periods of years. One idea that is frequently emphasized is that every ethnic culture is special, unique, and different. At a more general level, it is believed that music is not a universal language. Rather, it is assumed that it is more like a spoken language, a system of understanding that is learned only over an extended period of time. Similarly, understanding the unique features of musical cultures requires patient learning, not only of the sounds and rhythms, but also the ideas and beliefs related to music. The results of these studies show fascinating differences between music in different cultural contexts.

In the present study we adopt a cognitive approach, and examine in more detail the abilities that listeners from different cultural backgrounds have for understanding the basic structures of different musical traditions. One motivation for the present study is that, to the extent that this question has been studied in behavioral experiments, the differences obtained are not as large as might be expected. Two such cross-cultural studies (Castellano, Bharucha \& Krumhansl, 1984; Krumhansl, 1995b), described in more detail later, examined Chinese folk music and North Indian classical music. The former was a study of melodic expectancy, which is also the focus of the present study; the latter was a study of tonal hierarchies. These studies included listeners who were either from the music's cultural context or were from a Western culture. The results of both studies showed considerable agreement between the groups, and suggested that the inexperienced listeners were able to adapt quite rapidly to different musical systems. Moreover, they showed that similar underlying perceptual principles appear to operate, and that listeners are sensitive to statistical information in novel styles which gives important information about basic underlying structures.

The present study focuses on the topic of musical expectancy, specifically expectancy for melodic continuations. This focus was chosen because of the wealth of previous theoretical and empirical research on expectancy in music (see, for example, Carlsen, 1981; Carlsen, Divenyi \& Taylor, 1970; Cuddy \& Lunney, 1995; Jones, 1981, 1982, 1990; Jones \& Boltz, 1989; Krumhansl, 1995a,b, 1997; Schmuckler, 1989, 1990; Thompson, Cuddy \& Plaus, 1997; Unyk \& Carlsen, 1987). Expectancy is considered, at least in discussions of Western tonal music, to be a fundamental 
aspect of the music listening experience. It is presumed to play an important role in how listeners group the sounded events into coherent patterns and appreciate patterns of tension and relaxation. Expectations are assumed to exist on a variety of levels, including melodic, harmonic, rhythmic, and metrical, which may combine in complex ways giving music richness and variety. In addition to these functions, expectation may contribute to music's emotional effects as proposed by Meyer (1956) and Sloboda (1991).

Various theoretical models for expectancy have been offered, some of which are more narrowly based on principles of Western music and others which invoke more general psychological principles that may function across a range of musical styles. A proposal by Narmour (1990), called the implication-realization model, is notable for emphasizing the existence of general psychological principles (called bottom-up principles) that function independently of style knowledge. The model is most explicit in describing melodic expectations by providing precise, testable predictions. Cross-cultural studies offer a special opportunity for assessing the relative influence of general psychological principles, knowledge of the style and familiarity with the particular musical materials, and possibly also influences of knowledge of other styles inappropriately imposed on the musical materials.

In the present study, we investigate the basic cognitive processes underlying melodic systems in Sami yoiks. We take three approaches to studying expectancy in this style: a statistical style analysis of tones in a corpus of yoiks, a behavioral experiment, and a neural network model. Before describing the three approaches, we provide some background on the Sami yoiks and their cultural context. Although not all aspects of this description are directly relevant to the empirical research to be presented, we believe such background is an important component of music cognition in cross-cultural contexts.

\section{Sami yoiks: history and background}

The Sami people (sometimes referred to as Lapps) are indigenous people of the Scandinavian Peninsula (shown in Fig. 1, an area that is divided into Norway, Sweden, Finland, and Russia) and the Kola Peninsula (of present day Russia). They form a linguistic and cultural entity that is clearly distinguished from other peoples. Presently, it is believed that the Sami language developed from an early proto-Finnish language, and it is classified as a Finno-Ugric language. At the same time, various customs, traditions, languages, and dialects divide the Sami people into different subgroups. The size of the Sami population is estimated at somewhere between 60000 and 100 000. The different estimates depend on the emphasis given to various factors, including use of Sami language, adherence to the Sami way of life, and blood heritage. The Sami people's original source of food came from hunting and fishing. The earliest evidence of a specialized form of tame reindeer herding comes from the sixteenth century. It gradually became the most important livelihood, having more social status than hunting or fishing.

The Sami people and their culture have been an object of wide interest for some 


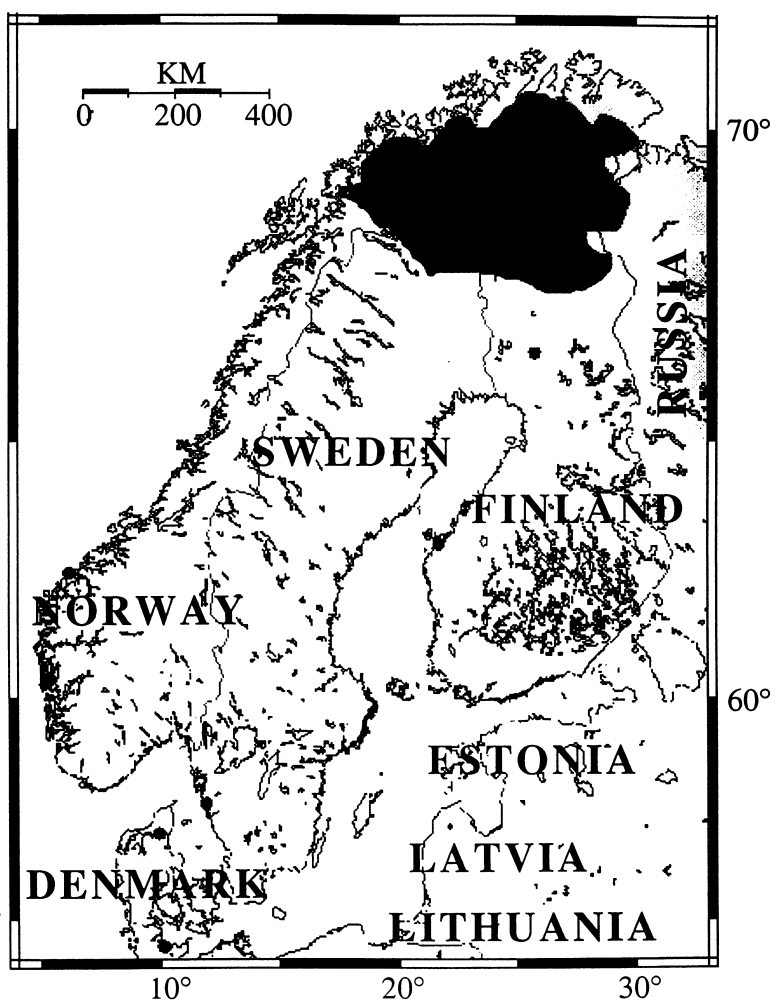

Fig. 1. Map of Northern Scandinavia showing North and Enare Sami regions.

centuries. Social, economic and religious circumstances have been written about since the seventeenth century, primarily by Christian missionaries from Sweden and Norway. Their writings about Sami music, and especially about yoik, suggest intolerance. Yoik was regarded as 'heathenism' or 'cursed songs', and only a few writers remarked positively about yoik as Sami poetry. The opposition was based in part on the old shamanistic belief that the melodies were given by the spirits (which survives partially to the present; Edström, 1978, p. 191).

The German-born Johannes Schefferus included two song texts by a Sami student Olaus Mattiae Sirma in his book 'Lapponia' which he published in 1673. The first transcriptions of yoik melodies were not published until the nineteenth century, among others by the Italian musician and traveler Giuseppe Acerbi and the German-born composer Johann Christian Friedrich Haeffner (1759-1833). Haeffner described a Sami performance in Stockholm in the 1780s in his 1818 'Anmärkningar öfver gamla nordiska sången' (for the transcription see, for example, Kjellström, Ternhag \& Rydving, 1988, pp. 112-113). Haeffner's descriptions of yoik's improvisatory character, its spontaneity, and connectedness to the context are notable. The great interest in folk music and folk songs in Sweden and Europe at that time did not extend to yoiks which remained only something 'exotic'. More conscious effort to 
collect yoik texts was made some decades later by the Finn Jacob Fellman (17951875), who worked as a parish priest in Utsjoki and collected many yoik texts and stories from that region during the 1820s.

The invention of the phonograph at the beginning of the twentieth century made serious scientific research on yoiks possible. A Finnish composer Armas Launis (1884-1959) traveled with his phonograph in the summers of 1904 and 1905 in the Lappmarks (inhabitance regions of the Samis) of Finland and Norway. He collected more than 800 melodies and melody variants, many of them from the yoiking of the famous Jouni Aikio (1875-1956), better known as 'Kaapin Jouni'. Launis (1908) published most of his results as a comparative study entitled 'Lappische Jouigos-Melodien'. The Swedish rail officer Karl Tirén (1869-1955) was a pioneer in Swedish yoik research. He traveled in Swedish Lappland in the 1910s, recorded approximately 700 melodies with his phonograph, and notated approximately 500 pieces (Tirén, 1942). In 1953 and 1954, the Sami scientist and herding school inspector Israel Ruong (1903-1986), together with Matts Arnberg and Håkan Unsgaard from the Swedish Broadcasting Company, traveled in Northern Sweden recording and documenting yoik material. The results were originally published as a set of records with an accompanying book, and recently issued as a pack of three CDs and a book (Arnberg, Ruong \& Unsgaard, 1969). In Sweden, the following, whose work is cited elsewhere in this section, should also be noted: Edström (1978) and Kjellström et al. (1988). Norwegians started their yoik documentation in the 1950s (Bakke, 1958), first at the museum and later at the University of Tromssa. Currently, Tromssa is also the center for a large-scale international research and documentation project on the Kola Sami music. In Finland, scholarly dissertations and articles have been published on yoiks and/or Sami music (for example, Jouste, 1998; Kantola, 1984; Saastamoinen, 1987, 1994, 1998). Some non-Scandinavian ethnomusicologists and researchers have also studied yoik and Sami culture (for example, Danckert, 1956; Lüderwaldt, 1976; Stockmann, 1986, 1987, 1990).

Sami music is almost exclusively vocal and, in addition to the yoiks, includes Lutheran hymns and other spiritual or profane songs either in Sami dialects or other languages. Most yoiks, especially in the Western and Northern Sami regions, are short miniature-like descriptions with few words and predominantly syllables without linguistic meaning. However, especially in Eastern regions, one finds some long epic poetry in yoik texts and even prose-like stories, which are performed in yoikmanner (sung rather than told). An instrumental music tradition is nearly non-existent because it is difficult to find materials for making instruments at these latitudes. The drum, often associated with Lappland, was primarily the instrument of the 'noaidi' (medicine man or shaman) for communicating with the otherworld, and was not meant for musical purposes. Dance music is equally sparse, although the Scolt Sami have a round dance tradition (Häkämies, 1978, cited by Kjellström et al., 1988).

The Sami people use many terms for singing with or without words, and their terminology is somewhat ambiguous and it varies across different regions. The key words are 'juoi'gat', 'juoiggus', 'juoigan' and 'luohti' in the North Sami dialect. The 
English word 'yoik' can be regarded as derived from these. The Sami verb 'juoi'gat' refers to the Sami way of singing a traditional Sami melody (Edström, 1978, p. 191). The term 'juoi' gat' can also be interpreted as 'to sing without words' (Arnberg et al., 1969, p. 7). 'Juoiggus' refers to the performance situation, its relationships between those present, body language, and so on. The case is similar with the word 'juoigan', 'the action of yoiking'. What is sung when someone yoiks is called 'luohti' (in some dialects also 'vuolle' and 'vuelie').

Traditionally, Sami yoiked when people gathered who knew each other well and shared memories. They recalled past experiences and yoiked each other. Mikkel Isak Oskal (Jernsletten, 1978, p. 111) recalls, "When I come to some place and they yoik me I know they welcome me kindly and accept me as one of them." Thus, an important function is to make someone feel that he or she belongs to the family and society, thus strengthening both the individual's and society's identity. The yoiks can also recall people, living or dead, who are not present. Mikkel Gaup, a Sami author and tradition bearer, remembers: "By yoiking my parents I can experience the feeling of being together with them, although they do not live anymore." (Eriksson, 1986, after Gaup). The message contained in yoiks (often with very few, sometimes only symbolic, words) is revealed at the time to only a small group of 'insiders' who share the same background and experiences (Jernsletten, 1978, pp. 110-111).

It was equally common to yoik in one's solitude. One basic reason for this was, of course, the natural desire for self-expression. Another reason was that the church, missionaries, Laestadianism (a religious movement), and many school teachers condemned yoiking (see, for example, Arnberg et al., 1969, p. 74; or Jernsletten, 1978, p. 113). A further reason was that in solitude one could feel someone else's presence through yoiking. Anders Baer puts it this way, "A luohti [yoik] is like a friend; when you are alone out in the mountains and remember a good friend, you yoik his luohti and it makes you feel good." (Jernsletten, 1978, p. 113). Thus, yoik is a way of expressing the feelings of togetherness beyond time and place and is at the same time both individual and collective.

The functions of yoik are quite different from Western music. According to Valkeapää (1984),

The yoik is not merely music. Its functions are much wider than that. They include ways to social contact. To calm down the reindeers. To frighten the wolves. The yoik was never intended to be performed as art. Art requires public. The yoik was used to call up friends, even enemies. The land and the environment. Animals. The yoik was also a step to another world, which makes it religious. What about its technique? If one compares yoik with Western music, it is soon obvious they are of different languages with different functions (Valkeapää, 1984, pp. 45-47, translation from Swedish by Pekka Toivanen).

Yoiks mostly deal with everyday life, and always have a personal meaning for the yoiker. Very seldom do the texts deal with general matters. The yoik describes something special from the yoiker's viewpoint and conveys his or her feelings. It 
can be irony, love, suffering, jealousy, sorrow, happiness, and so on. Therefore, yoik always has an object. Four main categories of yoik content can be identified: human beings, livelihoods, animals, and nature (Kjellström et al., 1988, p. 21). In addition, there are yoiks in which elements from multiple motif groups are interwoven to form a whole (Arnberg et al., 1969, p. 15).

The concrete and personal aspect of yoiking is seen most plainly in yoiks about people. Yoiks about human beings ('person yoiks') form a special art of remembering others. The primary objects are the closest relatives (parents, children, grandparents, etc.), but one could also yoik non-relatives, good friends, even enemies. Certain 'higher personalities', such as the members of the royalty, governors, officials, priests and doctors, could also be given their own yoiks. Traditionally it was considered improper to yoik one's own yoik (Kjellström et al., 1988, pp. 52-67). Yoiks about love, birth, death and other episodes in the course of human life can be counted in this category, as can yoiks about mythical figures in Sami stories.

In the yoik repertory dealing with livelihoods, those about reindeer herding form the main corpus. This may be due to the fact that it is precisely among the herders (the Sami who do the reindeer herding) that yoiking has traditionally had its most profound base (Valkeapää, 1984, p. 45). Another reason may be the high status of reindeer herding in Sami culture, an attractive theme to put into yoik. The relationship between man and animal, long wanderings in the fjeld (mountain) region, the actual work and its details, all these and more can be described in yoiks. In addition to reindeer, domestic animals, such as dogs, horses and goats, also have their own yoiks. Among wild animals, bear and wolf are the most popular objects in yoiks. Bear is yoiked in a very distinctive way, and its role in hunting ceremonies is similar to other Finno-Ugric people. Of wild birds the most important is the snow grouse. It is believed that the snow grouse has concealed all the wisdom of time underneath its wings.

Nature plays a significant role in the life of the Sami people. As a consequence, there are many yoiks about fjeldtops (mountain tops), valleys, forests, the sea, lakes, rivers, and the earth in general. Every region has its landmarks that play a special role in its inhabitants' life. Many of those landmarks have been considered to be sacred, thus adding a religious dimension to the yoik repertory. For example, a 'saivo' (a deep small lake with subterranean outlets) was considered to be a gateway to the 'other world' and a place where the deceased relatives continued their life. Naturally, as part of nature, the powers of weather (wind, thunder, clouds, rain, snow) have been described in yoiks, likewise the elements of space, such as the sun, moon, stars, Northern lights, and nightly darkness (Kjellström et al., 1988, pp. 44-52; see also Arnberg et al., 1969, pp. 17-21).

In recent years, traditional themes have yielded to more contemporary topics. A new yoik repertory has developed together with increased consciousness of the Sami identity as an indigenous people, gains in civil rights, the use of Sami language in education, and protection of the environment in order to maintain their way of life (including the traditional livelihoods). The songs of performers such as Mari Boine or Nils-Aslak Valkeapää have described various conflicts between the interests of the Sami people and the majority population. These new yoiks sometimes mix 
traditional elements of yoik with elements of contemporary popular and world music. For example, the use of rock instrumentation, or the use of instruments from European and non-European music cultures have, in some cases, replaced the traditional solo vocal performances. As with many musical traditions, a tension exists between preserving the music as something authentic and developing it as a living tradition.

Academic literature on Sami music from a music-theoretic point of view is still scant. Musically there are differences between yoiks of different regions. The following is a short summary of musical characteristics of North Sami yoiks used in this study, although these characteristics are found in other yoik regions as well. When, as in the case of yoik, music is learned and absorbed without a system of music theory or notation, and without instruction by master musicians, a large amount of variety and improvisation in performance is inevitable, either intended or unintended. An unexpected change in rhythmic cycle, vocal timbre, or mode during a performance is a yoiker's way of demonstrating his or her musical skill. It is difficult to say to what extent the precise musical events in a yoik performance are consciously planned.

Melodically, the (North Sami) yoik is characterized by short, repeated motives. These melodic motives often comprise only a few notes, in most cases from the major pentatonic scale. The fact that the motives contain only a few notes does not necessarily imply a narrow tonal range. On the contrary, the North Sami yoik's tonal range is often more than one octave. This is partly due to another feature of the yoik style, namely large interval leaps as will be discussed later. Saastamoinen refers to yoik's 'cyclic structure' as one of its most characteristic features. By 'cyclic' he refers to its lack of set beginnings and endings (Saastamoinen, 1994, pp. 28-30). A yoik can thus have more or less distinctive points, such as repeated melodic and rhythmic motives, intervals (very often fourths, either ascending, or descending), highs note or leaps, and 'chaining' questions and answers (a kind of 'call' and 'response'). If the rhythmic cycle is long, it distinguishes the individual performer. If the cycle is short, even small details take on a significant role. A single exceptional pitch or a single change in rhythmic pattern stands out from its musical environment. The long rhythmic cycles in many yoiks are not based on thinking of rhythm in beats of twos and threes, but rather thinking generally in terms of 'smaller' and 'larger' durations (Saastamoinen, 1994). It may be that this 'binarism' affects other musical elements of the yoik, for example, the 'higher' and the 'lower' in melody construction.

An interesting feature in yoik performances is the performer's tendency to raise the pitch level over time. Arnberg et al. (1969) suggest that this kind of phenomenon is emotionally conditioned. A person may start to yoik somewhat cautiously and begin rather low. The growing certainty and engagement then leads to a rise in both dynamics and pitch. This happens in both long narrative poems and in short repetitive luohtus and vuolles (Arnberg et al., 1969, p. 53). One of the authors (Pekka Toivanen) recalls a case where he (together with two other musicians) accompanied a yoiker at a music festival some years ago. Even in the presence of amplified instruments, the yoiker gradually raised the pitch during the performance of several 
yoiks. This phenomenon has also been documented by acoustic analyses (Johan Sundberg, pers. commun., 1998), which showed increases in F0 (fundamental frequency) of $20-40 \mathrm{~Hz}$ in less than a minute.

\section{Statistical style analysis of tones in a corpus of yoiks}

Although in recent years ethnomusicology has shown little interest in statistical analysis, there is in fact a long tradition of statistical studies in the past. Its roots are in the first half of this century in the research done, for example, by Densmore and Herzog (Ellingson, 1992, p. 131). Their work was continued and extended by the research of Freeman and Merriam (1956) and later by Lomax (1968). One objective of this approach was to describe and classify music, for example, by the statistical distribution of different intervals, rhythmic patterns, or pitches. In Finland, one program of research (described in more detail below) has been conducted in which similar statistical descriptions of yoik melodies were obtained (Kantola, 1984).

Music psychology has directed some attention to how statistical information affects, for example, perception and learning. This has been done using both cross-cultural studies, for example, with North Indian (Castellano et al., 1984) and Balinese (Kessler, Hansen \& Shepard, 1984) music, as well as with melodies in which the statistical properties of music were intentionally manipulated (Oram \& Cuddy, 1995). The results show that listeners are sensitive to pitch distributional information. It appears that for inexperienced listeners the pitch distributional information gives cues about the basic melodic and tonal structure of the music. In addition, it has been found that composers and improvisers emphasize the important tones of the tonality by using them more frequently, with longer duration, and more often in structurally important places than other tones (see Huron, 1993; Järvinen, 1995, 1997; Knopoff \& Hutchinson, 1983; Krumhansl, 1990). In addition, Thompson and Stainton (1998) showed that statistical distributions of tone sequences supported proposals concerning principles underlying melody formation (Narmour, 1990).

In the present study, statistical style analysis was employed in order to get precise information about yoik melodies. ${ }^{1}$ Our goal was different than the one motivating the previous statistical studies in ethnomusicology. In particular, we were interested in determining if there are some statistical regularities in the melodies that contribute to listeners' expectations, and whether these depend on their familiarity with the style. The musical corpus used consisted of 18 yoiks from the North Sami region. They are listed in Table 1 . The majority of this yoik corpus stems from the repertories of three well-known North Sami yoikers, Inga Eira Juuso (Kautokeino, Norway), Mattis Heatta (Kautokeino) and Wimme Saari (Enontekiö, Finland). In choosing these melodies we wanted to present a wide range of stylistic musical

\footnotetext{
${ }^{1}$ The music was analyzed with the Humdrum Toolkit (Huron, 1994) and some additional perl and shell scripts. These scripts can be found at http://www.jyu.fi/ tjarvine.
} 
Table 1

The yoiks used in the statistical style analysis of tones

\begin{aligned} & \hline$* 1$. Anden Inga (Inga Eira Juuso) \\ &$* 2$. Elle Sunna (Inga Eira Juuso) \\ &$* 3$. Cappa Magdalena (Inga Eira Juuso) \\ &$* 4$. Haldí (Wimme Saari) \\ &$* 5$. Bierra Bierra (Inga Eira Juuso) \\ &$* 6$. Val'gon guoi'ka (Mattis Heatta) \\ &$* 7$. Áilen Niga Elle (Mattis Heatta) \\ &$* 8$. Lemmon Elle (Mattis Heatta) \\ & 9. Asla Piera (Mattis Heatta) \\ & 10. Anda Niilas (Mattis Heatta) \\ & 11. Homman Lasse (Mattis Heatta) \\ & 12. Bear's Yoik (Wimme Saari) \\ & 13. Turra-Biedde (Wimme Saari) \\ & 14. Dansés Lille Sara (Inga Eira Juuso) \\ & 15. 'Once I tried reindeer-herding' (public domain) \\ & 16. 'Helgetlagan bielloskálla' (public domain) \\ & 17. Molkesvuomi Silba (Mattis Heatta) \\ & 18. Illu ja Moras (Mattis Heatta) \\ & \hline\end{aligned}

features of the North Sami area. The statistical distribution of the tones in the corpus of 18 yoiks was determined, that is, the frequency with which tones occur. In addition, we investigated the frequencies of two- and three-tone transitions. The subset of materials actually used in the behavioral experiment, indicated by asterisks, was also examined separately and the results were generally similar, so the following discussion presents only the results from the larger corpus.

\subsection{Results of the statistical style analysis}

All the yoiks were transposed so that they fit in the range from the $\mathrm{G}$ below middle $\mathrm{C}$ (denoted G) to the e an octave and a third above middle $\mathrm{C}$ (denoted $\mathrm{e}^{\prime}$ ). They were transposed in such a way that $\mathrm{C}$ appeared relatively frequently. The top of Fig. 2 illustrates the distribution of tone onsets in the transposed yoiks expressed in proportions. The values were collapsed across octaves and are shown as a function of pitch class. The tones C, D, E, and G are clearly used most frequently, and the other tones are used infrequently. These tones imply a pentatonic scale, although the low frequency of the tone A should be noted. The high frequency of both $\mathrm{C}$ and $\mathrm{G}$ is interesting, because it suggests that there are two relatively strong reference tones in the yoiks included in this study. The bottom of Fig. 2 illustrates the distribution of the tones without making the data octave equivalent, that is, over the full range of 22 tones. It shows that the most frequently used tone is middle $\mathrm{C}$. Thus, the frequent use of the tone G seen at the top of Fig. 2 appears to be a result of the use of this tone in both octaves ( $\mathrm{G}$ and $\mathrm{g}$ ).

The relatively frequent use of the second (D) and third (E) scale degrees may imply stepwise melodic motion above middle $\mathrm{C}$ which further emphasizes its position as a reference tone. The low frequency of the tone $\mathrm{B}$ below middle $\mathrm{C}$ also 

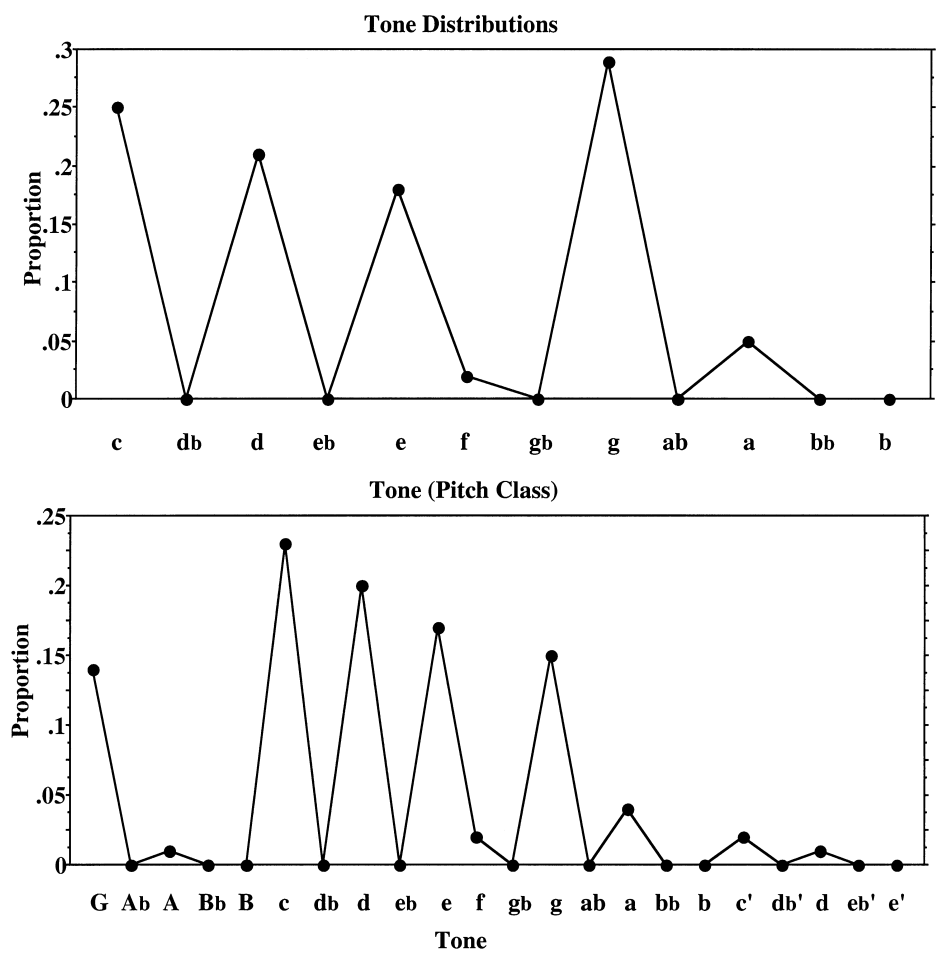

Fig. 2. The tone distributions for the yoiks in the statistical style analysis. At the top, the data are collapsed into a one-octave range. At the bottom, the data are shown over the full 22-tone range. Capital letters denote tones in the octave below middle $\mathrm{C}$. Letters with primes indicate tones in the octave beginning with the high $\mathrm{C}$.

implies that these yoik melodies do not have cadential motion like Western tonal music, however. The tone $\mathrm{G}$ is preceded and followed in most cases by an interval jump, because the tones $A$ and $F$ are used infrequently. Although the tone $G$ is not emphasized by stepwise melodic motion, an analysis of the phrase structure showed that $\mathrm{G}$ had a very prominent role in the melodies. In particular, most phrases begin with the tone $\mathrm{G}$ and end with either $\mathrm{G}$ or $\mathrm{C}$. Therefore, it seems plausible that both $\mathrm{G}$ and $\mathrm{C}$ can be considered reference tones.

In addition to tone onsets, two- and three-tone transitions were also obtained from the yoik melodies. Table 2 shows the two-tone transitions that were found and the frequency with which they occurred. (The three-tone transitions are given in Appendix A.) The transition matrix shows that wide interval leaps are quite common, and these are often the consonant intervals of perfect fourths and fifths (e.g. G c, d g, c g, $\mathrm{Gd}$ ). Thus, most of the larger numbers in the tables that are far from the diagonal of the matrix in Table 2 correspond to consonant intervals. Also, for any given tone there are many different possible continuations. These transitions also give supporting evidence for the interpretations given above to the tone-frequency profiles. For example, for the tone $\mathrm{G}$ in the lower octave, the most common transition is to $\mathrm{c}$ 


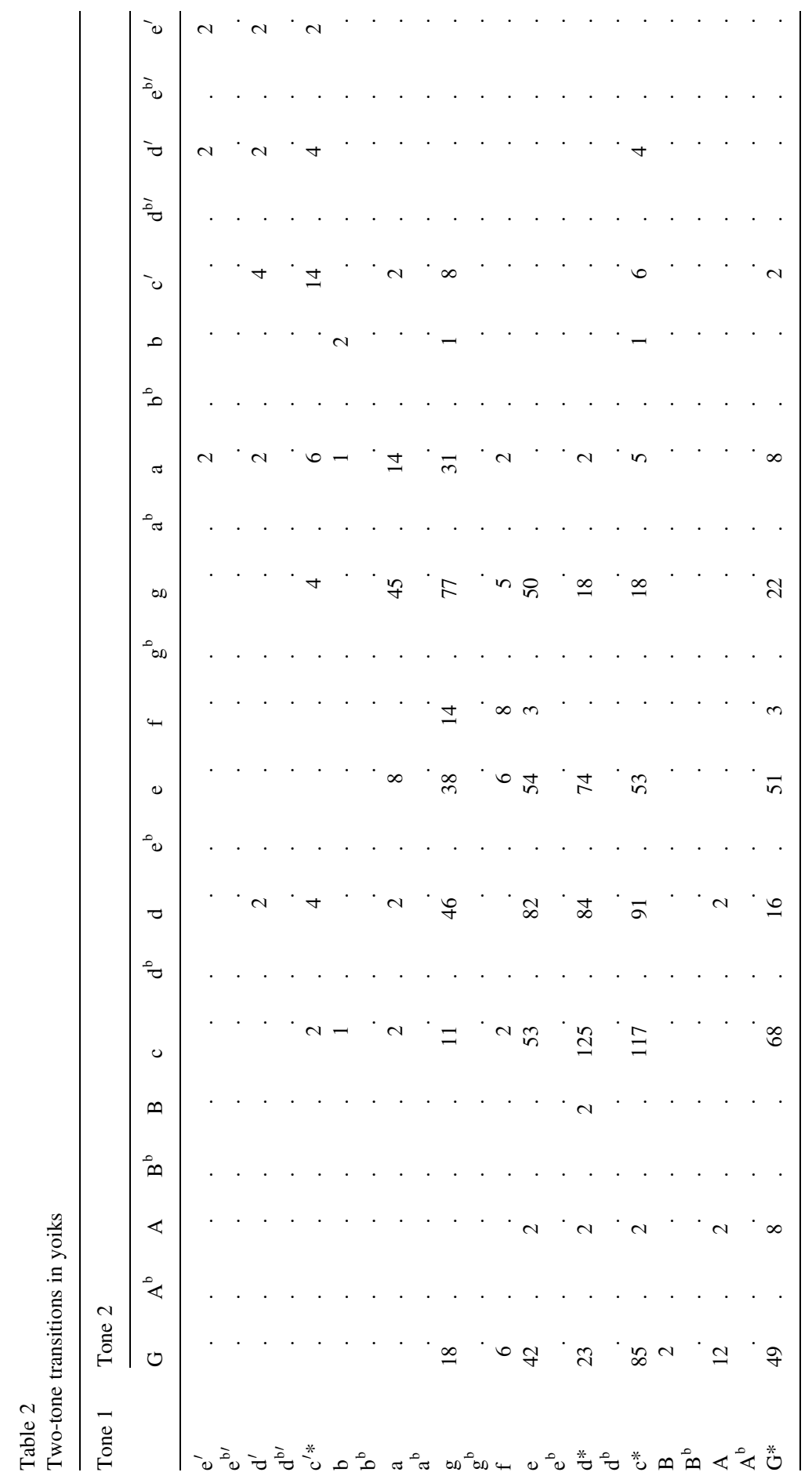


(middle C). It is noteworthy that the transition from A to $\mathrm{G}$ occurs relatively infrequently, so $\mathrm{G}$ is not emphasized by step-wise motion. The most common transition in the whole set is from $d$ to $c$ consistent with the idea that the tone $d$ is used in stepwise melodic motion to c. Furthermore, the tone B is never followed by the tone c. This observation again suggests that these yoik melodies do not use B as a way of establishing $\mathrm{c}$ as a single central reference tone. Thus, in various ways these results show that the tonal organization does not comply with principles of Western tonal music.

The statistical study of yoiks by Kantola (1984) produced similar results to those obtained here. Her corpus consisted of 154 yoiks from the Northern (Tana) Sami area. The statistical analysis indicated that the pentatonic scale predominated, representing $71 \%$ of the repertoire; the diatonic scale was found in $7 \%$ of the repertoire, and the tones consisting of the first five diatonic scale tones $(\mathrm{C}, \mathrm{D}, \mathrm{E}, \mathrm{F}, \mathrm{G})$ was found in $6 \%$ of the repertoire. It was possible to reconstruct tone distributions from her report for a total of 903 tones. This distribution correlated highly $(r(10)=0.96$, $P<0.001$ ) with the distribution in the present study (the values shown at the top of Fig. 2). Very similar distributions of two-tone transitions (intervals) were also found. In both her corpus and the present corpus, a high proportion of two-tone transitions consisted of relatively small intervals of a major third or less ( 74 and $60 \%$, respectively). However, perfect fourths were common in both (14 and 20\%, respectively). Perfect fifths also appeared fairly frequently ( 5 and $8 \%$, respectively). When the two sets of intervals were made comparable (by combining intervals of a major third or less, which was Kantola's procedure), the interval distributions were almost identical $(r(7)=0.99, P<0.001)$. Thus, Kantola's statistical analysis of tones in another, larger corpus of yoiks converged on the major features identified in the present statistical style analysis of tones.

\section{Behavioral experiment}

Previous psychological experiments on melodic expectancy (e.g. Carlsen, 1981; Carlsen et al., 1970; Cuddy \& Lunney, 1995; Krumhansl, 1991, 1995a,b, 1997; Schmuckler, 1989, 1990; Thompson et al., 1997; Unyk \& Carlsen, 1987) have focused on tone-to-tone expectancy, as is the case here. Essentially all of these studies have been done with Western listeners and, where musical excerpts have been used, they have been drawn from Western music. An exception to this is one experiment in which excerpts of Chinese folk music were presented to native Chinese and Western listeners (Krumhansl, 1995b). These were followed by different possible continuation tones which listeners rated in terms of how well they fit with their expectations for continuation. The two groups of listeners gave very similar results. In addition, similar underlying psychological principles were suggested when the data were analyzed within the framework of the Narmour (1990) implication-realization model, to be described in more detail below. The listeners' judgments were most strongly influenced by proximity, a preference for tones close to the final tone of the musical excerpts. The data also supported the 
model's principles concerning the direction of the melodic interval, the size of the interval, proximity to the second to last tone of the excerpt, closure (a preference for a large interval to be followed by a smaller interval and reversal of direction), and pentatonic scale. The relative weights of these principles in predicting the responses were similar for the two groups of listeners.

Another cross-cultural study examined hierarchies of tones in classical North Indian music, again finding remarkable consistency between (South Asian) Indian and Western listeners (Castellano et al., 1984). Excerpts from ragas were followed by tones that were judged in terms of how well they fit with the context. Both groups of listeners gave higher ratings to the tones theoretically predicted to be important structural tones. These tones were also sounded most frequently and for the longest total duration. The hierarchy was headed by the Sa, followed by the $\mathrm{Pa}$, followed by the vadi tone of the rag, and finally the other scale (that) tones. Indian listeners were somewhat more sensitive to scale membership, whereas Western listeners showed more hierarchical differentiation among the scale tones. It is notable that, in this study, Western listeners were able to suppress tonal hierarchies applicable to Western music; their responses did not correspond to tonal hierarchies of major and minor keys but rather to theoretical predictions for Indian music.

Another study should be mentioned that was conducted in parallel with the present study and used the same methods (Krumhansl, Louhivuori, Toiviainen, Järvinen \& Eerola, 1999). The corpus of music in that case consisted of Finnish spiritual folk hymns. The musical practice concerning these hymns is that it is learned by members of the Beseecher religious movement from family members and other members of the sect. The hymns are not widely known by other Finns. This allowed us to obtain behavioral responses from listeners with generally similar cultural and musical backgrounds. However, one group of listeners from the Beseecher community, called experts, had extensive knowledge of the musical style and was familiar with the particular materials used in the experiment. This difference between groups produced interpretable patterns in melodic continuation judgments that could be traced to stylistic features uncovered through both ethnomusicological studies and statistical style analyses. The melodic continuation ratings for the experts (compared with non-experts) reflected more strongly expectations based on detailed knowledge of the style, specifically three-tone transitions and the tone that actually occurs next in the hymn. The opposite was true for more general or schematic expectations, such as those found in studies using two-tone contexts and schematic key-defining contexts (Krumhansl, 1995a; Krumhansl \& Kessler, 1982). These Western schematic influences were stronger for the non-experts than the experts.

The Krumhansl (1995a) study will also be used in the analysis of the present results. In that study, two-tone contexts were followed by a third tone which listeners rated in terms of how well it fit with their expectations for how the interval would continue. This study was done with Western listeners and showed that even the minimal two-tone contexts produced quite consistent and interpretable judgments of continuation tones. (Studies in which continuations were performed by subjects - 
sung or played on a keyboard - show similar results; Carlsen, 1981; Carlsen et al., 1970; Schmuckler, 1990; Thompson et al., 1997; Unyk \& Carlsen, 1987.) The strongest influence on these data was tonality, particularly the tonal hierarchies of the major and minor keys (Krumhansl \& Kessler, 1982) that contain the context interval. Thus, the two-tone context intervals are apparently sufficient for Western listeners to invoke a rich set of expectations within a system of interrelated keys. These data will be taken here as representative of general, schematic Western expectations. In addition, that study found an influence of consonance and general support for the principles of the Narmour (1990) implication-realization model.

The results of the present experiment will also be tested to see how well they conform to predictions of the implication-realization model (Narmour, 1990). The tests will consider the model as originally formulated and numerically coded (Krumhansl, 1991, 1995b), as modified in response to subsequent data (Krumhansl, 1995a), and as simplified through reanalysis of previous data (Schellenberg, 1996, 1997). These articles, the article by Krumhansl et al. (1999), and Appendix B should be consulted for more technical aspects of the model. Briefly, Narmour (1990) proposed five underlying principles that generate a system of melodic archetypes. These principles apply to points in music when expectation for continuation is strong, that is, where the melody sounds incomplete. The last two tones at such points form what is called the implicative interval; the last tone of the implicative interval and the tone that follows is called the realized interval. The five principles describe how the melody is expected to continue, i.e. what realized interval is expected given the implicative interval. The expectations depend on the size of the implicative interval and its direction.

Two principles are governed by proximity; they state that a tone will be expected if it is close to the second tone of the implicative interval (Proximity, PR) or the first tone of the implicative interval (Registral Return, RR). The third principle (Registral Direction, RD) is governed by good continuation; it states that a tone will be expected if the implicative interval is a small interval and the realized interval continues in the same direction, or if the implicative interval is large and the realized interval reverses the direction. The fourth principle (Closure, CL) states that a sense of closure is induced when the melody changes direction and when a large interval is followed by a smaller interval. The fifth principle (Intervallic Difference, ID) is governed by similarity; it states that if the implicative interval is small a similarly-sized realized interval is expected, whereas if the implicative interval is large a smaller realized interval is expected.

These principles are precisely specified in the implication-realization model in terms of interval size. For example, the principle of Proximity (in the original formulation in Narmour, 1990) states that the strength of expectation decreases with interval size down to six semitones (a tritone) and then remains at a constant low level. Registral Return is satisfied when the first tone of the implicative interval and the last tone of the realized interval are two semitones or less (a major second or smaller). This interval precision permits quantitative predictions that can be tested against behavioral data (Krumhansl, 1991, 1995b). In previous research, the quantified model was tested using melodic judgments made for contexts drawn from 
English folk songs, Chinese folk songs, and atonal songs (Krumhansl, 1995b), finding statistical support for most of the principles. Evidence for the principles also comes from studies by Cuddy and Lunney (1995), and Thompson et al. (1997), and a statistical style analysis by Thompson and Stainton (1998) of Bohemian folk song melodies. Krumhansl (1997) found evidence that the principles also operate for non-adjacent tones on a higher hierarchical level, in addition to the toneto-tone level.

Subsequent research has considered both additions to the model and simplifications of the model as originally quantified. A better fit to the data of Krumhansl (1995a), described above, was found by a modified model that coded Proximity and Registral Return as linearly decreasing functions of interval size (without cut-off points at six and three semitones, respectively). This coding of Proximity and Registral Return is shown in Appendix B. These data also suggested that the model needed to be augmented by including effects of consonance. Specifically, a variable coding consonance was constructed for the realized interval and for the interval formed by the first tone of the implicative interval and the second tone of the realized interval. It used empirical and theoretical values of consonance summarized in Krumhansl (1990, p. 57). Specifically, the values used were the average of the normalized values of the set of empirical and theoretical values, shown in Appendix B. The Krumhansl (1995a) study also suggested the addition of variables coding unisons and octaves of the realized interval. The negative weight found for unisons indicates that repeated tones are not expected and the positive weight for octaves means that octaves are more expected than the other principles would predict. This revised model, including the originally formulated principles of Registral Direction, Intervallic Difference, and Closure (see Appendix B), provided a better fit to the earlier data using contexts from English folk songs, Chinese folk songs, and atonal songs, and also the two-tone context data of Cuddy and Lunney (1995).

Schellenberg (1996) reanalyzed the data for the English folk songs, Chinese folk songs, and atonal songs (Krumhansl, 1995b), leading to a revised and simplified model. He proposed coding Proximity as a linear function (as had Krumhansl, 1995a) and a new coding of Registral Direction that assumed the principle only applies to intervals a perfect fifth or larger. Registral Return was coded as originally. He also omitted Intervallic Difference and Closure from the model. This simplified model provided an improved fit to the data of the three styles of music over the original formulation (Krumhansl, 1995b), but a worse fit for the Chinese folk songs and atonal songs compared to Krumhansl (1995a). The original coding also provided a slightly better fit to the production data of Carlsen (1981) and Unyk and Carlsen (1987) than the revised model of Schellenberg (1996).

In a subsequent reanalysis of the same melodic continuation data, Schellenberg (1997) proposed a further modification. It combined the principles of Registral Return and Registral Direction into a single principle, called Pitch Reversal. This, together with the linearly coded Proximity principle, constitutes his two-factor model. It again performed better than the original formulation (Krumhansl, 
1995b) on all three styles of music, but worse on the Chinese folk songs and atonal songs compared to Krumhansl (1995a). Repeated analysis of the of same data carries the risk of modeling specific details of the results that do not extend more generally. Consequently, the data from the present experiment will be used to test the various proposed extensions and simplifications of the implication-realization model.

To sum, three themes that emerge from these studies will be important here. The first is the possibility that listeners are quite flexible in responding to musical structure in unfamiliar styles and are largely able to suppress style-inappropriate expectations. The second is that the distribution of tones in the music may orient naive listeners to the appropriate tonal organization. The third is the possibility that a set of underlying psychological principles exists that apply across styles. The behavioral data can be used to test different formulations of the principles of the implication-realization model (Narmour, 1990) and determine their relative weights, which may vary across cultural contexts. The test materials for the behavioral experiment were a subset of eight of the yoiks listed in Table 1, indicated by asterisks. Fig. 3 shows a complete yoik in which the context that was excerpted from the yoik for the perceptual experiment is indicated by brackets. The context was followed by different possible continuation tones at the position marked PP ('probe position'), which listeners were to judge in terms of how well they fit with their expectation. Fig. 4 shows the seven other contexts used in the experiment together with the correct continuation tones. The probe positions were selected intuitively to coincide with points where expectancy for continuation would be strong. Many of them have unexpected leaps in the melody at the probe position. The basis for selecting these probe positions is given in more detail in Table 3 for each of the yoiks.

\subsection{Method of the behavioral experiment}

\subsubsection{Participants}

Three groups of listeners participated. The first group were native Sami, some from the North Sami region and some from the Enare Sami region (see Fig. 1). They

Val'gon guoi'ka (No 6, complete)

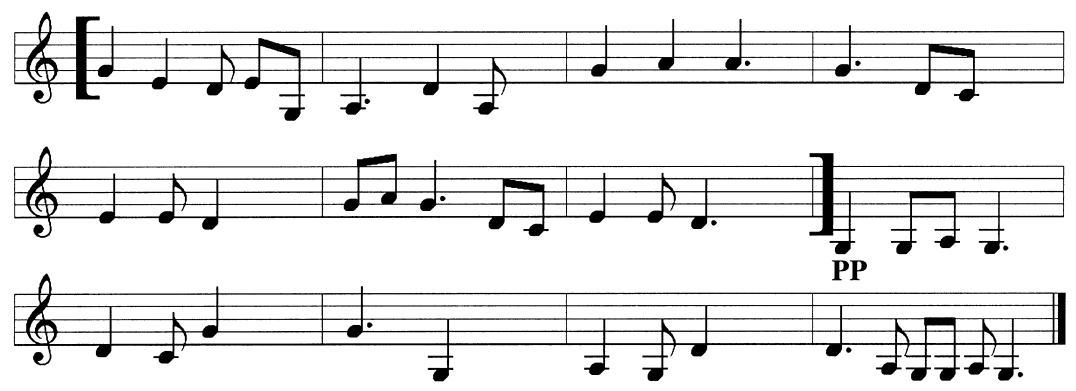

Fig. 3. Example of a complete yoik melody, 'Val'gon guoi'ka'. The brackets show the section selected as a test excerpt. The probe position is indicated by (PP). 

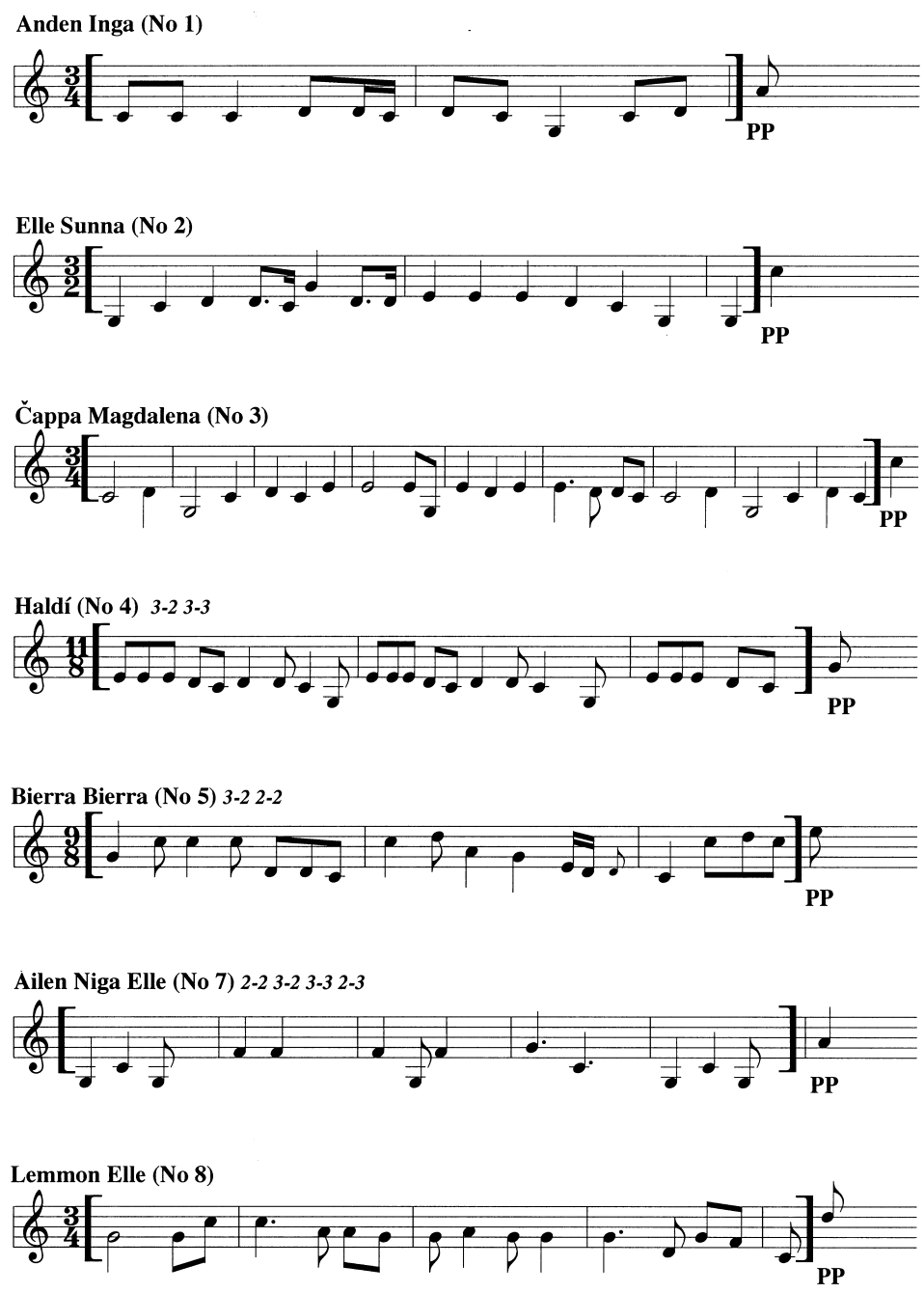

Fig. 4. The other seven excerpts used in the experiment; the probe positions are indicated by (PP).

had extensive experience learning and performing yoiks. ${ }^{2}$ Due to difficulties in getting these experts together, the experts were tested individually on separate occasions (in Helsinki and Jyväskylä, Finland, and in Kautokeino, which is the center of Sami culture in Northern Norway). They were paid a small compensation. All experts were born and grew up in Sami culture and had extensive experience

\footnotetext{
${ }^{2}$ Originally 14 Sami listeners were tested. Seven of them, who were students in the Kautokeino Sami High School, were unable to complete the experiment because of a change in class schedule. None of these listeners were active yoikers. Therefore, only the data of the seven experts who completed the full version are reported in this study.
} 
Table 3

Selection of probe positions in yoiks

1. Anden Inga: the large interval leap to a tone that is new in the context. The tone $g$ might have been expected given the $\mathrm{G} \mathrm{c}$ d context.

2. Elle Sunna: the very wide interval leap outside the previous range.

3. Cappa Magdalena: the large interval leap outside the previous range.

4. Haldí: the change from the repeated pattern. The tone $\mathrm{G}$ would have been expected.

5. Bierra Bierra: the preceding wide interval leap from $\mathrm{c}$ to $\mathrm{c}^{\prime} \mathrm{d}^{\prime} \mathrm{c}^{\prime}$ would create expectations for a note that fills the gap.

6. Val'gon guoi'ka: the larger interval leap downward to $\mathrm{G}$ which widens the range. The tone c would have been expected.

7. Áilen Niga Elle: the large interval leap to a. The c might have been expected because it would have repeated the preceding pattern.

8. Lemmon Elle: the large leap outside the previously established range that appears on a weak beat.

with yoiks. The expert subjects' age range was from 22 to 39 years (average 30.0 years). Three of them were men, four women. They had participated in musical activities for an average of 13.7 years and had listened to yoiks extensively and also performed yoiks in public. They had formal music training on average for 4.3 years and two of them had studied some music theory and had played instruments. The majority of them (6/7) were musicians and four of them were well-known yoikers. ${ }^{3}$ The experts reported they were highly familiar with this musical style. The average rating of familiarity with the style was 6.8 on a scale from 1 to 7 , where 7 is maximal familiarity. However, their familiarity with the individual yoiks used in the experiment (as opposed to the style in general) varied (see Table 4). As can be seen, they were very familiar with only two of the yoiks selected as experimental materials.

The 11 Finnish subjects were undergraduate (first and third year) music education majors from the Department of Music, University of Jyväskylä. Their primary training was in Western music. These students, however, were interesting for our purposes because they had learned to play a number of yoiks over a 1-year period and were familiar with two of the yoiks used in the experiment. The average age of the students was 21.6 years (range 19-24 years). They had participated in musical activities on average for 14.9 years, had studied music professionally on average for 2.0 years, and had studied music theory on average for 8.8 years. The experiment was a part of their music course. They were familiar with some of the yoiks used in the experiment (particularly, yoiks 4 and 6, 'Haldí' and 'Val'gon guoi'ka', see Table 4) because they had been used in ethnomusicology and performance courses. The students had practiced a medley of 14 yoiks for about a year and performed them in at least four public concerts. The average rating of familiarity with the style in general was 4.0 on a scale from 1 to 7 , where 7 is maximal familiarity. The music listening habits and musical tastes of the two groups were similar except that the Sami subjects reported listening to popular Sami music whereas the nonexperts did not.

\footnotetext{
${ }^{3}$ Angelin Tytöt (Angel Nieiddat in Sami) (Tuuni and Ursula Länsman from Angeli village of Inari, Finland), Annukka Hirvasvuopio from Utsjoki, Finland, and Isak Vars from Kautokeino, Norway.
} 
Table 4

Familiarity with the Yoiks

\begin{tabular}{lll}
\hline Yoik & Experts & Non-experts \\
\hline Anden Inga & 6.3 & 1.6 \\
Elle Sunna & 2.4 & 1.4 \\
Cappa Magdalena & 2.3 & 1.5 \\
Haldí & 3.0 & 6.9 \\
Bierra Bierra & 6.1 & 1.7 \\
Val'gon guoi'ka & 2.1 & 6.7 \\
Áilen Niga Elle & 3.7 & 3.2 \\
Lemmon Elle & 2.1 & 1.1 \\
\hline
\end{tabular}

The third group consisted of 21 subjects who were participants in The Intensive Program in Cognitive Musicology, IPCM (held in Jyväskylä, Finland, August, 1998). They were from various countries in Europe and their average age was 26.1 years (range 22-49 years). They had participated in musical activities on average for 17.3 years, had studied music professionally on average for 4.8 years, and had studied music theory on average for 4.7 years; $95 \%$ of them had not heard any yoiks before and they were not familiar with the yoik melodies (the average familiarity rating with the style of this group of subjects was 2.1).

\subsubsection{Apparatus and stimulus materials}

The music was recorded on tape by playing a synthesizer (Ensoniq EPS 16 Plus keyboard synthesizer with a sampling rate $44.1 \mathrm{kHz}, 16$-bit resolution) under computer control. Using a synthesizer was necessary, not only for producing the experimental materials with sufficient control, but also for pitch extraction in the computational model described later. In particular, the rise in pitch that is typical of yoik performances, noted above, prevented us from using recorded performances. For the experiment, the tapes were replayed by either a stereo or a high-quality portable cassette player. The 18 yoiks were first transposed so that they fit into the range from $\mathrm{G}$ (the $\mathrm{G}$ below middle $\mathrm{C}$ ) to $\mathrm{e}^{\prime}$ (the e an octave and a third above middle C). They were then performed on keyboard by Pekka Toivanen and recorded in MIDI. Therefore, the performance contained improvisatory aspects that are typical of the yoik style, such as short ornamental tones. The experimental materials were then derived from the MIDI files. The experimental trials consisted of the context plus an additional continuation tone. The continuation tones were the 22 chromatic scale tones in the range from $\mathrm{G}$ to $\mathrm{e}^{\prime}$. The continuation tones were produced by manipulating the original next pitch in the yoik, therefore keeping the rhythm and dynamic the same as the original next tone. The trials were reproduced with an English horn sample, which had an amplitude envelope close to a sung human voice.

\subsubsection{Procedure}

The listeners were given the instructions (in Sami, Finnish or English) in written form and the instructions were also read and explained to them. They were told that they would hear fragments of melodies that start at the beginning of a phrase and 
stop in the middle of a phrase. They were asked to rate how well the continuation tone fitted with their expectations about what might follow in the melody. The rating was made on a scale from 1 ('extremely bad continuation') to 7 ('extremely good continuation'). These ratings will be called fitness judgments. After a set of five practice trials, the actual experiment began with a block of trials for each melodic context. At the beginning of each block of trials, the context was presented three times so that listeners could become familiar with it and rate how familiar they were with the yoik (on a scale from 1 to 7 ) prior to the experiment. A 10-s break was then followed by 24 trials. The first two were for practice and had the same continuation tones as the last two trials; these were followed by the 22 experimental trials corresponding to each of the possible continuation tones. In each block of trials, the order of the continuation tones was random. The experiment lasted about $60 \mathrm{~min}$ including the instructions and the practice trials. The Western and Finnish subjects were tested as groups, and the Sami subjects were tested individually or in a group of two or three. The procedure was otherwise the same for the groups.

All subjects marked their responses on answer sheets, and at the end of the experiment they filled out a questionnaire about their musical backgrounds. Contrary to our initial methodological concerns, none of the participants exhibited difficulty adapting to the experimental task. In particular, the timbre of the synthesizer appeared to present no problem for the Sami, possibly because they were all familiar with popular music. Moreover, one of the yoikers remarked enthusiastically how useful the information from the experiment would be to him as a performer.

\subsection{Results of the behavioral experiment}

\subsubsection{Intersubject and intergroup correlations}

The fitness judgments of the continuation tones were consistent between subjects within groups. The average intersubject correlations within groups were $r(174)=0.53,0.72$, and 0.51 for the Sami, Finnish, and Western subjects, respectively (all $P<0.0001$ ). Because of the significant within-group correlations, the average results will be used in most further analyses of the data. After the data were averaged for each group, the intergroup correlations were computed. They were $r(174)=0.87$ between the Sami and Finnish subjects, 0.82 between the Sami and Western subjects, and 0.91 between the Finnish and Western subjects. These correlations suggest that the Finnish subjects were intermediate between the Sami and Western subjects. Although many of the following analyses focus on differences between groups, these should be viewed within the context of these relatively high intergroup correlations.

\subsubsection{Sample results}

Fig. 5 shows a representative set of results for one of the yoiks, Bierra Bierra, that was familiar to the Sami but not the other two groups of subjects. As can be seen, the correct next tone $\left(\mathrm{e}^{\prime}\right)$ received the highest rating by the Sami, lower ratings by the Finnish, and lowest ratings by the Western subjects. Fig. 6 shows a representative set of results for one of the yoiks, Val'gon guoi'ka, that was unfamiliar to the Sami and 


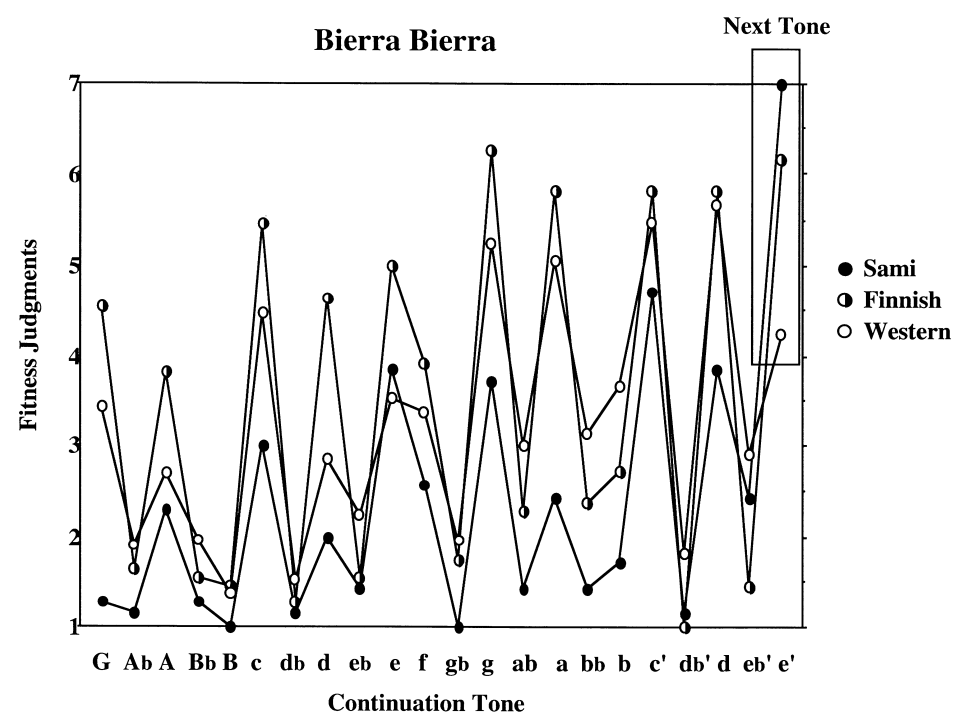

Fig. 5. The fitness judgments of Sami, Finnish, and Western listeners for the yoik 'Bierra bierra'. This yoik was familiar only to the Sami listeners.

Western subjects but was familiar to the Finnish subjects. The highest rating for the correct next tone $(\mathrm{G})$ was given by the Finnish subjects, followed closely by the Sami, and then the Western subjects. In general, the Sami and Finnish responses were very similar for this yoik $(r(174)=0.97, P<0.0001)$. This suggests that familiarity with the particular yoik can compensate for the lower experience of the Finnish subjects with the style compared with the Sami subjects. For both of these yoiks, lower ratings tended to be given to tones farther from the last tone of the context ( $c^{\prime}$ for Bierra Bierra and d for Val'gon guoi'ka).

For the yoiks in both Figs. 5 and 6, it is clear that subjects gave higher ratings to the tones of the pentatonic scale, C, D, E, G, and A, relative to their non-scale neighbors. Fig. 7 shows the fitness judgments given to all the continuation tones averaged across all the yoiks. The top graph shows the average responses for the octave-equivalent pitch classes. The bottom graph shows the average responses for the tones without collapsing across octaves. Comparison with Fig. 2 shows that the fitness judgments correspond quite closely with the frequency with which the tones are sounded in the yoiks. The following analyses are directed at studying these and other factors that influenced the three sets of data, beginning with bottom-up principles and then turning to top-down expectations.

\subsubsection{Bottom-up principles: the implication-realization model and consonance}

Four models, shown in Table 5, were tested against the data for each group, and also against the data for each subject. Model 1 coded the five principles of the implication-realization model as was done by Krumhansl (1995b). Model 2 coded the five principles of the implication-realization model as was done by Krumhansl 


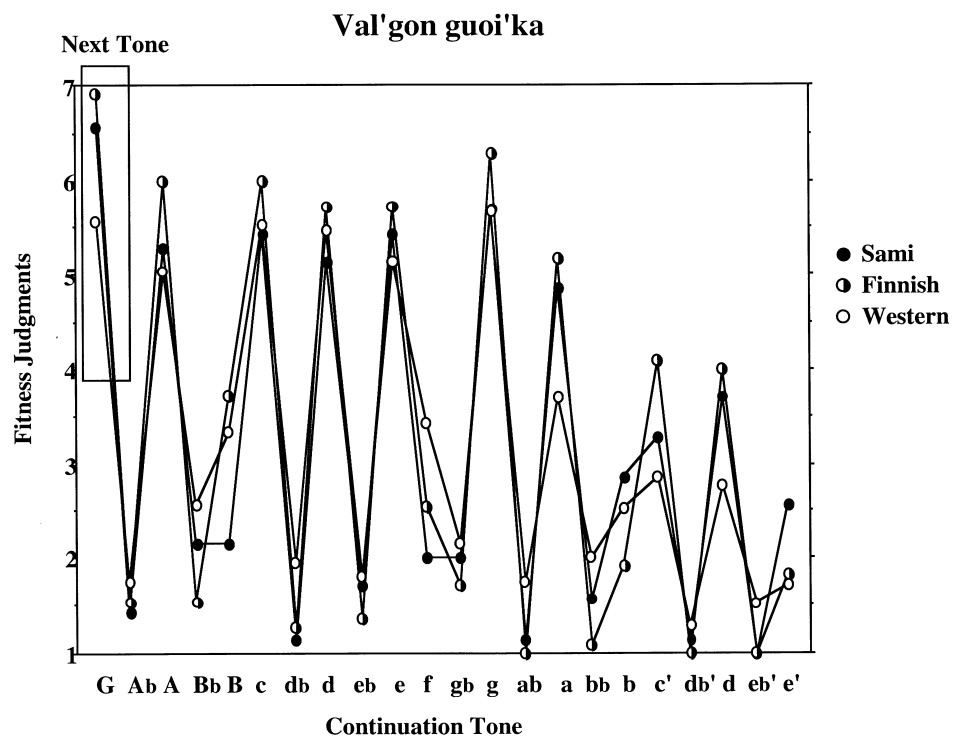

Fig. 6. The fitness judgments of Sami, Finnish, and Western listeners for the yoik 'Val'gon guoi'ka'. This yoik was familiar only to the Finnish listeners.

(1995a), with the linear codings of Proximity and Registral Return together with two consonance variables (see Appendix B). Model 3 coded three principles of the implication-realization model as was done in the revised model by Schellenberg (1996). Model 4 is the two-factor version of the implication-realization model proposed by Schellenberg (1997).

Table 5 shows the multiple correlations for the four models, all of which were significant, and the adjusted $R^{2}$ values. Model 2 out-performed the others which performed about equally to one another. To test for differences between models, the individual subjects' multiple correlations were entered into an analysis of variance with the result showing highly significant differences between the models $(F(3,114)=180.8, P<0.0001)$. This difference was that Model 2 provided a better fit than the other three models which were not significantly different from one another. Analyses for each model showed differences between the three groups of subjects. Models 1, 3, and 4 fit the data for the Western subjects better than the Sami and Finnish subjects $(F(2,36)=7.2,6.4$, and 6.4, for Models 1, 3, and 4, respectively, all significant at $P<0.01)$. The groups were not significantly different from one another for Model $2(F(2,36)=0.75, \mathrm{NS})$. Thus, the Narmour (1990) formulation of the model as originally coded by Krumhansl (1995b) and the revisions to the model by Schellenberg $(1996,1997)$ all appear to have a Western tonal bias. Not only did they perform best on the data from Western tonal music in previous studies, but they fit the data of Western listeners better than the Finnish and Sami listeners in this experiment.

Table 5 also shows which principles made significant contributions to the fit of the 

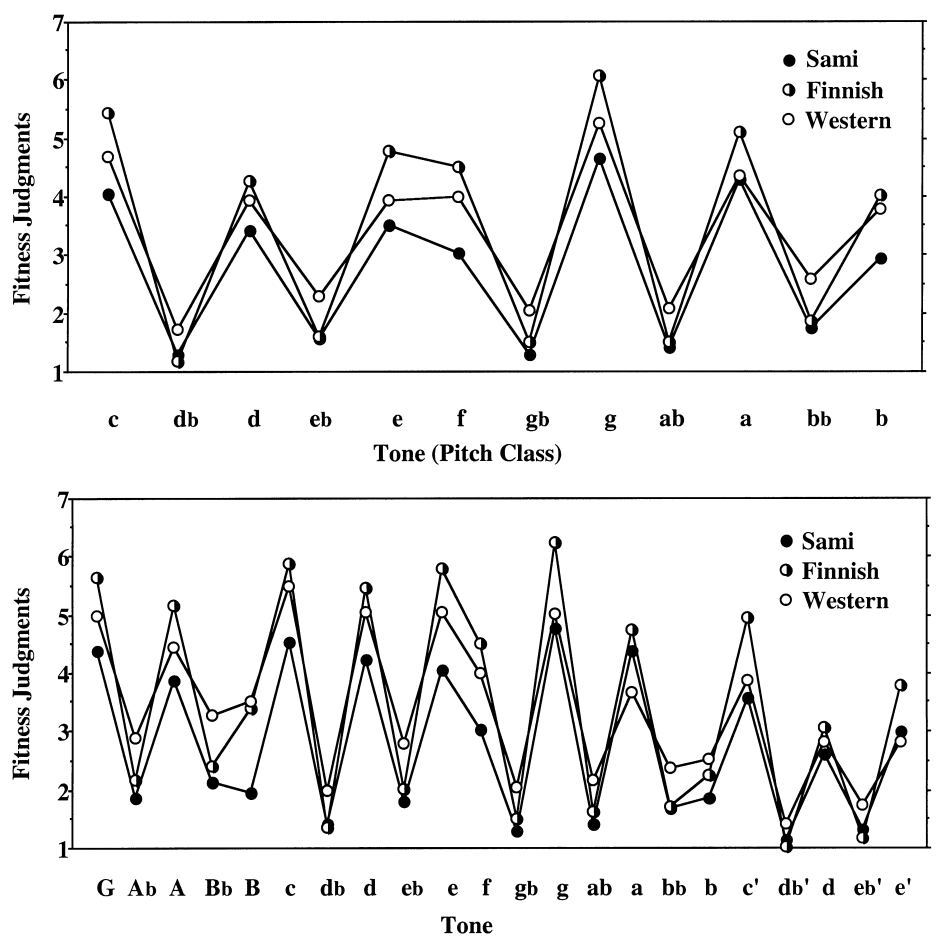

Fig. 7. The results of the behavioral experiment averaged across the eight yoik contexts for the two groups of listeners. At the top, the data are collapsed into a one-octave range. At the bottom, the data are shown over the full 22-tone range. Capital letters denote tones in the octave below middle C. Letters with primes indicate tones in the octave beginning with the high $\mathrm{C}$.

models. The large difference between models can be attributed to the highly significant effects of the two Consonance variables in Model 2. In addition, Intervallic Difference made significant contributions for Models 1 and 2, arguing against its elimination in Models 3 and 4 of Schellenberg (1996, 1997). For Models 3 and 4, only Proximity made a significant contribution (in the linear form). The proposed Pitch Reversal variable (Schellenberg, 1997) was not significant. The revised Registral Direction variable (Schellenberg, 1996) could not be tested because of zero variance for the cases included in the experiment. In sum, Model 2 substantially outperformed the other models so that in what follows, only Model 2 will be considered.

Fig. 8 shows the simple correlations between the seven variables of Model 2 and the data of the three groups of subjects. The graph, in which the dashed line indicates the correlation that is significant at $P<0.05$, generally confirms the results of the multiple regression. For all three groups of subjects, the importance of the two Consonance variables is seen as the high correlations at the right. In the simple correlations, Intervallic Difference, Registral Return (linear), and Proximity (linear) were relatively strong and significant. These were considerably stronger for the 
Table 5

Bottom-up principles: the implication-realization model and consonance

\begin{tabular}{|c|c|c|c|}
\hline & Sami & Finnish & Western \\
\hline \multicolumn{4}{|c|}{$\begin{array}{l}\text { Model 1: five implication-realization } \\
\quad \text { principles coded as in Krumhansl (1995b) }\end{array}$} \\
\hline$R(5,170)$ (adjusted $\left.R^{2}\right)$ & $0.29 *^{\mathrm{a}}(0.06)$ & $0.27 *(0.05)$ & $0.45 * * *(0.18)$ \\
\hline \multicolumn{4}{|l|}{ Registral Direction } \\
\hline Intervallic Difference & $* *$ & $* *$ & $* *$ \\
\hline Registral Return & & & $*$ \\
\hline \multicolumn{4}{|l|}{ Proximity } \\
\hline \multicolumn{4}{|l|}{ Closure } \\
\hline \multicolumn{4}{|c|}{$\begin{array}{l}\text { Model 2: five implication-realization } \\
\text { principles coded as in Krumhansl (1995a) } \\
\text { and consonance }\end{array}$} \\
\hline$R(7,168)$ (adjusted $\left.R^{2}\right)$ & $0.71 * * *(0.48)$ & $0.73 * * *(0.52)$ & $0.79 * * *(0.62)$ \\
\hline \multicolumn{4}{|l|}{ Registral Direction } \\
\hline Intervallic Difference & $*$ & $* *$ & $* *$ \\
\hline \multicolumn{4}{|l|}{ Registral Return (linear) } \\
\hline Proximity (linear) & & & $*$ \\
\hline \multicolumn{4}{|l|}{ Closure } \\
\hline Consonance (with last tone) & $* * *$ & $* * *$ & $* * *$ \\
\hline Consonance (with second to last tone) & $* * *$ & $* * *$ & $* * *$ \\
\hline \multicolumn{4}{|l|}{$\begin{array}{l}\text { Model 3: three implication-realization } \\
\text { principles coded as in Schellenberg (1996) }\end{array}$} \\
\hline$R(3,172)$ (adjusted $\left.R^{2}\right)$ & $0.27 * *(0.06)$ & $0.28 * * *(0.07)$ & $0.50 * * *(0.24)$ \\
\hline \multicolumn{4}{|l|}{ Registral Direction (revised) ${ }^{\mathrm{b}}$} \\
\hline \multicolumn{4}{|l|}{ Registral Return } \\
\hline Proximity (linear) & $* * *$ & $* * *$ & $* * *$ \\
\hline \multicolumn{4}{|l|}{ Model 4: two implication-realization } \\
\hline$R(2,173)$ (adjusted $\left.R^{2}\right)$ & $0.27 * *(0.06)$ & $0.28 * * *(0.07)$ & $0.50 * * *(0.24)$ \\
\hline \multicolumn{4}{|l|}{ Pitch Reversal } \\
\hline Proximity (linear) & $* * *$ & $* * *$ & $* * *$ \\
\hline
\end{tabular}

a $* P<0.05, * * P<0.01$, *** $P<0.001$.

${ }^{\mathrm{b}}$ Cannot test because of zero variance for the experimental materials.

Western subjects than the other two groups of subjects, which in part explains their relatively high multiple regressions for Models 1, 3, and 4 (each of which contain some of these variables) compared to the other subjects. Registral Direction and Closure were not significant for any of the groups.

To summarize the results in this section, the strongest bottom-up influence on the results overall was consonance. Listeners in all three groups gave higher ratings to continuation tones that formed a consonant interval with either the last tone or the second to last tone of the context. In addition, support was found for some of the principles of the implication-realization model, specifically Intervallic Difference and, to some extent, Proximity and Registral Return. Neither Registral Direction nor 


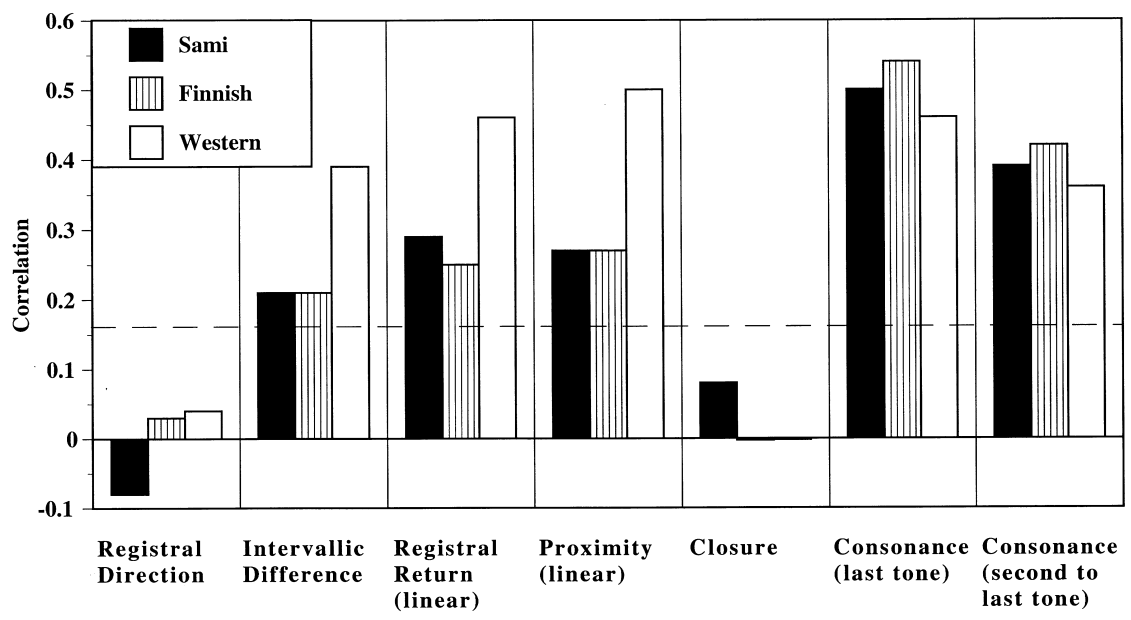

Fig. 8. The simple correlations between the behavioral data for Western, Finnish, and Sami listeners and the factors in Model 2. These include principles of the Narmour (1990) implication-realization model's principles (as modified by Krumhansl, 1995a) and consonance.

Closure were influential. These results support the addition of consonance to the implication-realization model (as suggested by Krumhansl, 1995a), but not the revisions proposed by Schellenberg $(1996,1997)$.

These results are interesting to compare with previous studies, especially that of Krumhansl et al. (1999). One group of listeners in that study consisted of Finnish music students, similar in background to the Finnish subjects in the present experiment. In the earlier study, the strongest influence came from the principle of Proximity, with some influence of Registral Return and Intervallic Difference; the influence of consonance was relatively weak. Thus, although the Finnish subjects in the two studies were similar in terms of their musical experience, consonance was the dominant factor only for the yoik contexts in the present study. Recall that the statistical style analysis, described earlier, found a relatively large number of consonant intervals. This suggests that listeners adapt their judgments to the intervals present in the experimental contexts. Additional comparisons with the statistical style analysis will be presented later. Despite the relatively weak influence of consonance in the earlier study, the model including this factor (Model 2) provided a better account of the data than the other formulations of the model. The difference between models in the earlier study was not as large as in the present case. As in the present study, no support was found for the revisions to the model proposed by Schellenberg $(1996,1997)$, suggesting that these revisions need to be reassessed in light of experimental data from a broader range of musical styles.

\subsubsection{Western schematic versus veridical expectations}

The two-tone context data (Krumhansl, 1995a) are taken here to be representative of Western schematic knowledge. The correlations between these judgments and those of the three groups of subjects are shown on the left of Fig. 9. The top graph is 
for all yoiks, the next graph is for the yoiks familiar to the Sami listeners but not the Finnish (or Western) listeners, the next graph is for the yoiks unfamiliar to the Sami (and Western) listeners but familiar to the Finnish listeners, and the bottom graph is for the yoiks unfamiliar to all three groups of listeners. As can be seen, the influence of schematic Western knowledge was greatest for the Western listeners, followed by the Finnish listeners, and finally the Sami listeners. The differences were particularly strong for the yoiks familiar to the Sami listeners but not the Finnish listeners, and were weakest for the yoiks familiar to the Finnish listeners but not the other groups.
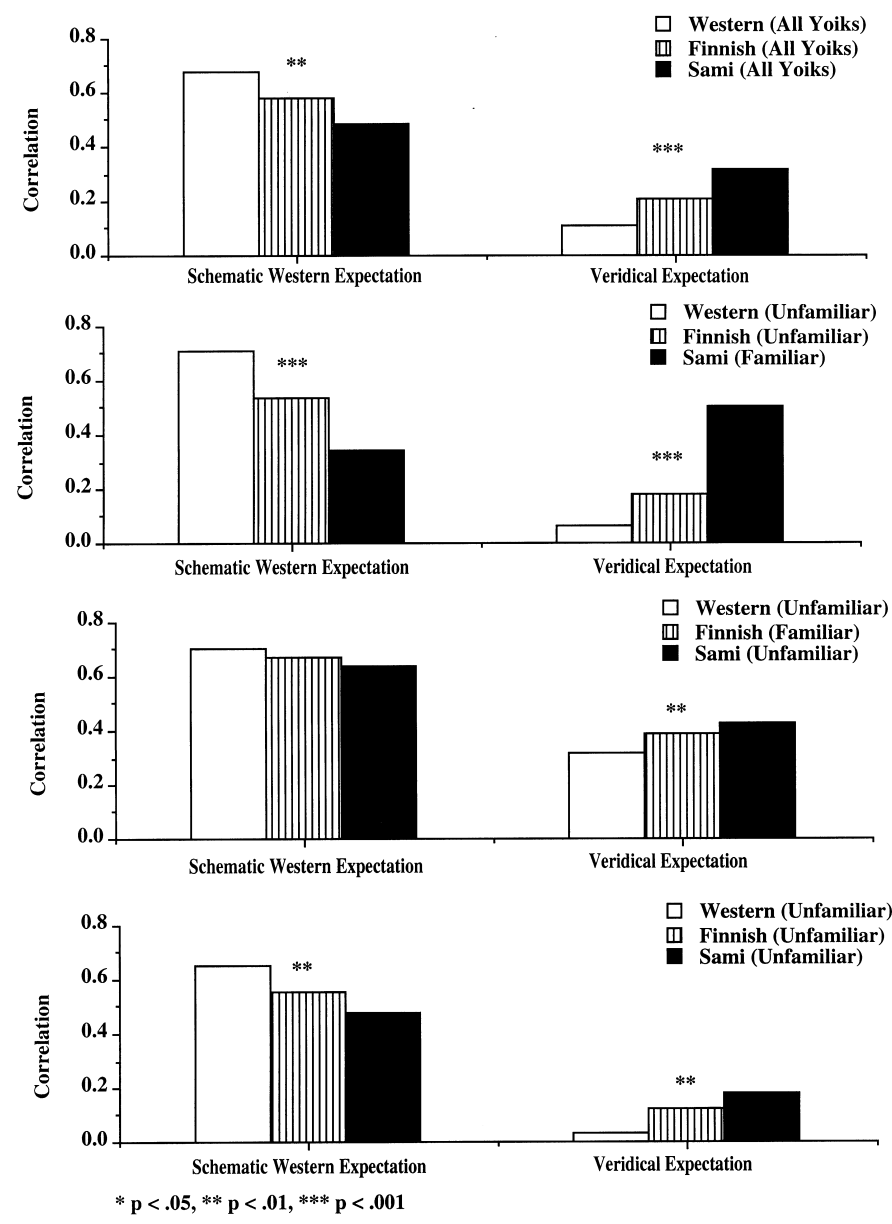

Fig. 9. The left shows the correlations between schematic Western expectation (as measured by Krumhansl, 1995a) and the fitness judgments made by Western, Finnish, and Sami listeners. The right shows the correlations between veridical expectation (correct next tone), and the fitness judgments made by Western, Finnish, and Sami listeners. The top graphs show the results for all yoiks, the second graph shows the results for the yoiks known only to the Sami listeners, the third graph shows the results for the yoiks known only to the Finnish listeners, and the bottom graph shows the results for the yoiks not known to any of the groups of listeners. 
Individual subject correlations with the two-tone context data (Krumhansl, 1995a) were computed to test for significant differences between groups. The resulting statistical tests for the four graphs were $F(2,36)=5.51, \quad P<0.01$, $F(2,36)=10.03, P<0.001, F(2,36)=3.04$, NS, and $F(2,36)=5.30, P<0.01$, respectively. Thus, experience with the music was inversely related to the influence of schematic knowledge of Western tonal-harmonic music as assessed in the twotone data of Krumhansl (1995a).

For veridical knowledge, the inverse pattern was found, as seen in the graphs on the right of Fig. 9. The influence of the correct next tone was strongest for the Sami subjects, followed by the Finnish subjects, and then the Western subjects $(F(2,36)=19.31, P<0.0001)$. Again, the difference between groups was largest for the yoiks that were familiar to the Sami subjects $(F(2,36)=27.60, P<0.0001)$, as seen in the second graph. The differences between groups were smaller for the yoiks that were familiar to the Finnish but not the Sami subjects $(F(2,36)=6.92$, $P<0.01)$, and the yoiks that were unfamiliar to all subjects $(F(2,36)=7.55$, $P<0.01)$. Because the correct next tone variable is highly skewed, the difference between groups was substantiated by an analysis of the judgments given to the correct next tone for all yoiks. The rating given the correct next tone was, on average, 2.2 points higher than their average rating for all tones for the Sami, compared to 1.8 points and 0.7 points for the Finnish and Western subjects, respectively $(F(2,36)=20.26, P<0.0001)$.

To summarize, these results show the patterns that would be expected. The judgments of Western subjects were most influenced by schematic Western knowledge, and the judgments of the Sami were least influenced by schematic Western knowledge. The results showed the complementary pattern for veridical knowledge, with the veridical expectations strongest for the Sami subjects. Moreover, these differences were related to prior knowledge of the particular yoiks, such that the differences were largest for the yoiks with which the Sami subjects were familiar. These differences that depended on style knowledge are similar to those found in the study of Finnish spiritual folk hymns (Krumhansl et al., 1999).

\section{SOM simulation}

The listeners' ratings of continuation tones are assumed to be based on a conceptual representation of structures typical of the relevant musical style. These representations have been suggested to develop through repeated exposure to music and the regularities inherent in it (Bharucha, 1987; Krumhansl, 1990; Oram \& Cuddy, 1995). In the present study, the development of such conceptual representations was simulated with a neural network model of the self-organizing type (Kohonen, 1997), denoted SOM and described in more detail below. In previous work, Bharucha and Todd (1989) modeled the formation of general tonal-harmonic expectancy (schematic expectancy) and expectancy for the correct continuation of a piece (veridical expectancy). For this, they used a sequential neural network based on the design by Jordan (1986). They showed that the network learned a novel tonal sequence more 
quickly if the sequence conformed to familiar patterns (specifically, frequent chord transitions) in previously learned sequences. Moreover, they showed that the networks were able to produce highly-expected transitions more quickly than unexpected ones. Louhivuori, Kaipainen and Toiviainen (1997) modeled the learning and production of Karelian rune songs with a modified SOM. After training with a set of rune songs, their model was able to produce new melodic variants of the learned songs.

The SOM is an artificial neural network that simulates the formation of ordered feature maps, such as sound frequency maps found in the brain. It consists of an input layer and a two-dimensional map layer. The latter consists of map units that, in a simplified way, imitate the function of biological neurons. All the input units are connected to all the map units with connections whose weights are adaptable. The self-organizing algorithm used for training the SOM is simple. First, an input is randomly selected from a set of inputs. Second, the winner unit, that is, the unit whose connection weights are closest to the input, is determined. Third, the connection weights are adapted so as to better match the input. The adaptation is strongest for the winner unit and weaker for units that are located far from the winner unit. This cycle is repeated a large number of times, typically on the order 100000 , with different inputs randomly selected for each cycle. During this process, the learning rate, determining the strength of the adaptation, and the neighborhood radius, determining the size of the area around the winner where adaptation is strong, are gradually decreased. This is sufficient for the map layer units to become responsive to input patterns in an orderly manner. The process of self-organization can be shown to be equivalent to minimizing the average quantization error for the set of input vectors (Kohonen, 1997, pp. 127-130), where quantization error is defined as the distance between the input vector and the closest reference vector on the map.

Once the network has been trained, the quantization error provides a measure of how well a given input fits with the generalized representation obtained from the training data. In other words, the network can then be probed to see how closely it matches the behavioral judgments of melodic expectancy. This is done in the following way. When an input is presented to the SOM, it evokes an activation pattern. The closer the connection weights of a unit are to the input, the higher the unit's activation value. The highest activation value on the map determines the closest reference vector. This distance from the input vector to the closest reference vector, the quantization error, provides a measure of how well this input fits with what the SOM has learned. Input vectors typical of the musical style learned by the map would have a small quantization error, and vice versa. Consequently, the goodnessof-fit of a given sequence to the SOM, called the fitness measure, was defined as the opposite of the quantization error of the input vector for the melodic sequence. In this way, the SOM can be tested to see if it exhibits the same response structure as the listeners' melodic continuation judgments.

The input data used in the simulations consisted of acoustic representations of the yoiks. In order to use the data as input to the SOM, it was necessary to preprocess the sound files to extract significant features. Because the focus was on modeling melody perception, the most essential feature of the tones to be extracted was 
pitch. The melodies were monophonic, so this could be carried out using a relatively simple algorithm based on periodicity analysis. Briefly, the periodicity analysis was carried out by a set of autocorrelation units that calculate the production of the actual input signal and the same signal shifted backwards in time by varying time-lags. For more technical details about the pitch extraction algorithm used see Krumhansl et al. (1999).

Like most neural networks, the basic SOM is inherently static in the sense that it is not capable of processing sequential input. Because the temporal order of tones is central to melody perception, the order should be represented in the model in some way. One way of dealing with this is to convert sequential information into static form by constructing a set of memory traces and using them for training the SOM. In the present study, the memory traces were constructed using a diffusion mechanism. This mechanism weights recent events more strongly and represents them with a finer temporal precision than less recent events. A mathematical description of the diffusion memory algorithm used in the present study is given in Krumhansl et al. (1999). The preprocessing stage, which is depicted in Fig. 10, yielded as output a 132-dimensional vector every $40 \mathrm{~ms}$ representing the melodic progression during the last few seconds. This period agrees with what is known about the length of auditory sensory memory, also known as the echoic store or long-term sensory store (Cowan, 1988, 1995). Typical estimates of the length of this store are in the range between about 2 and 5 s (e.g. Darwin, Turvey \& Crowder, 1972; Fraisse, 1982; Treisman, 1964). Further, the memory span used in the present simulations also agrees with temporal integration periods used in other simulations of similar type (e.g. Huron \& Parncutt, 1993; Leman, 1995).

Two different corpora of melodies were used for training the SOM. One corpus consisted of the eight yoik contexts used in the behavioral experiment, including the correct continuation tone. The SOM trained with this corpus will be called the Yoik SOM and this SOM should model detailed properties of the musical style, including the correct next tone in the melody. The second corpus consisted of eight Finnish melodies. Four were Lutheran hymns (two in major and two in minor), and four were Finnish folk songs (two in major and two in minor). ${ }^{4}$ This corpus was chosen to be familiar to the Finnish subjects, and so will be called the Finnish SOM. However, these melodies would also be stylistically familiar to the Western subjects because both hymns and folk songs have properties that are typical of European melodies. Nonetheless, we will call this the Finnish SOM for consistency with Krumhansl et al. (1999) in which it was also used.

To train the SOM, the input sound file was first preprocessed using pitch extraction and diffusion memory, as described above. In each of the simulations, the SOM

\footnotetext{
${ }^{4}$ The Lutheran hymns were Enkeli taivaan (Angel of heaven), Martin Luther, 1535 (hymn book number 21, as designated by the Finnish Evangelic Lutheran church in 1986), Jumala ompi linnamme (God is our fortress), Martin Luther, 1528 (hymn book number 170), Lienenkö outo (Am I a stranger?), German, 1675 (hymn book number 281), and Vakaana Herran teitä (Steady on the Lord's path), Johan Olof Wallin, 1816 (hymn book number 393). The Finnish folk songs were Minä olen Härmän Kankaanpäästä (I am from Kankaanpää, Härmä), Ol' kaunis kesäilta (It was a beautiful summer evening), Aamulla varhain (Early in the morning), and Taivas on sininen ja valkoinen (The sky is blue and white).
} 


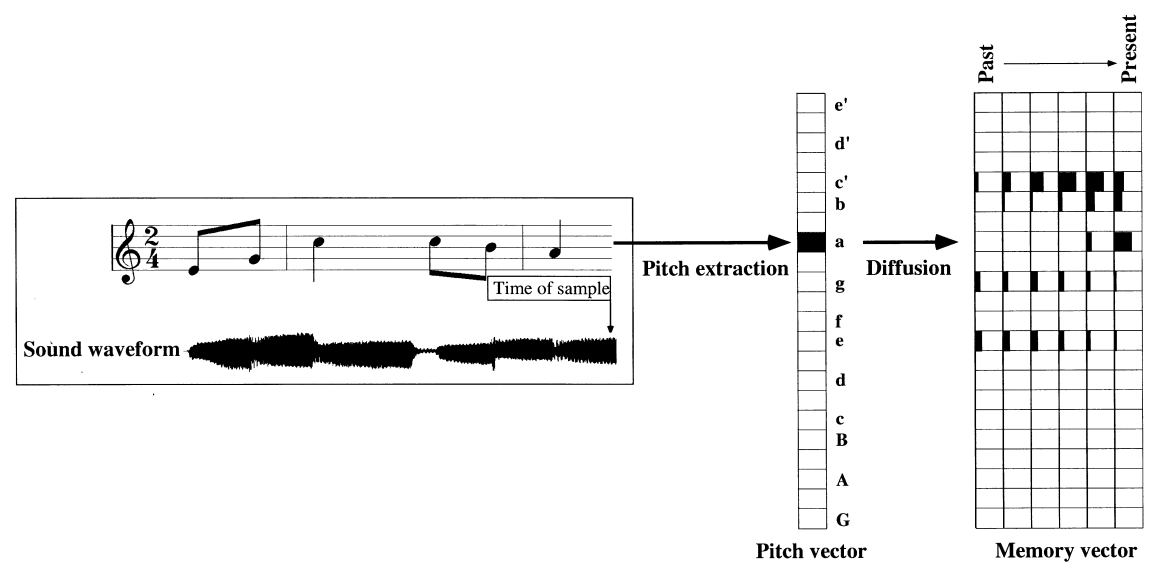

Fig. 10. Preprocessing of the input data for the SOM. The pitch and memory vectors shown in the figure were obtained at the point of time indicated on the sound waveform image. At this point, pitch a is sounded. The component of the pitch vector representing this pitch has a high value, whereas all the other components have zero values. In the memory vector, the most recent pitches are represented in the rightmost column. This column contains a high value at pitch a and somewhat lower values at pitches $\mathrm{c}^{\prime}$ and $\mathrm{b}$. Correspondingly, the oldest events of the memory span are represented in the leftmost column. This column contains the highest value at pitch e and lower values at pitches $g$ and $c^{\prime}$.

consisted of 30 by 30 units, the size suggested by previous tests as a good compromise between the resolution of the network and the time needed for training. The training was carried out in two phases. The ordering phase consisted of 10000 cycles, where each cycle consisted of choosing a vector randomly from the set of input vectors and adapting the SOM to this vector. During the ordering phase, the learning rate was linearly decreased from 0.50 to 0.10 . Similarly, the neighborhood radius was decreased linearly from 15 to 3 , where the unit of measure is the distance between two adjacent units on the map. The convergence phase consisted of 500000 cycles, during which the learning rate was linearly decreased from 0.10 to 0 and the neighborhood radius from 3 to 0 . After the SOM was trained, its response to each of the experimental sequences was measured. For this, the sound files containing the sequences were preprocessed as described above. For each test melody, the input used for testing the SOM was the diffusion memory trace taken at the end of the sequence. For each memory trace, the fitness measure was determined.

\subsection{Results of the SOM simulation}

\subsubsection{Correlations with the behavioral data}

Fig. 11 shows the average correlations between the fitness measures of the two SOMs and the average behavioral data from all three groups of subjects. The top graphs shows the correlations for all yoiks, the next graphs show those for the yoiks familiar to the Sami subjects but not the others, the next graph shows those for the yoiks familiar to the Finnish subjects but not the others, and the bottom graph shows 

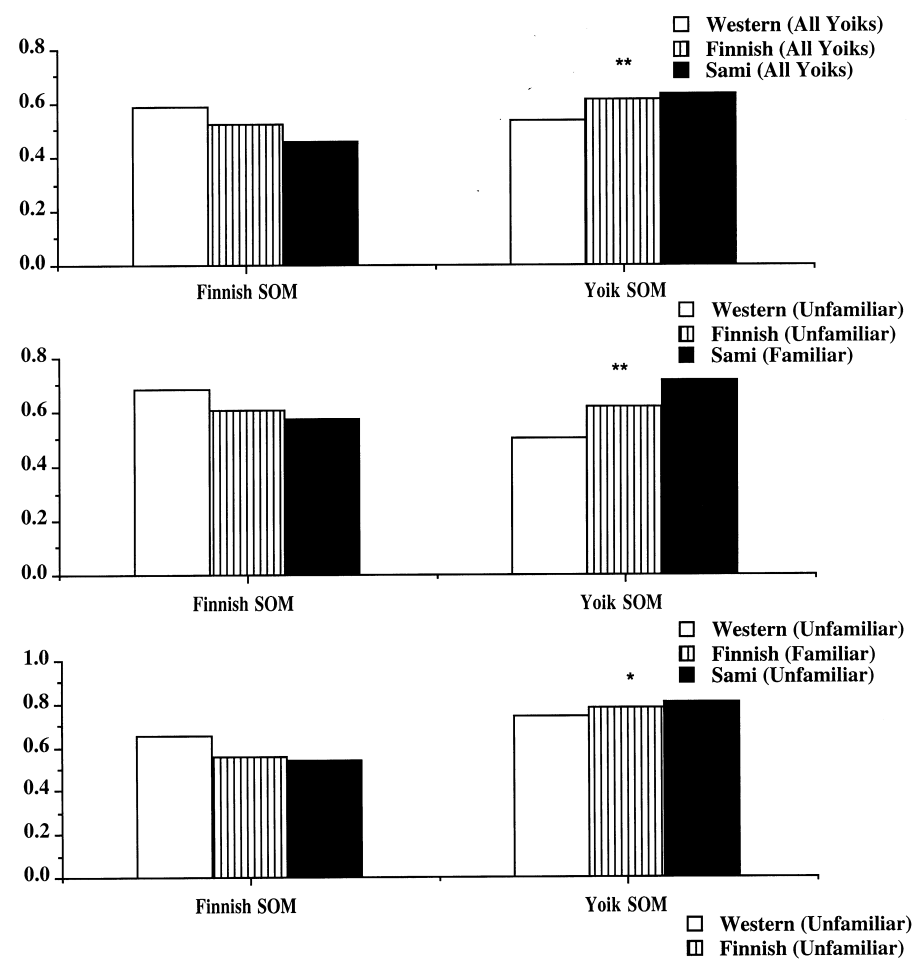

* $\quad$ I Finnish (Unfamiliar)

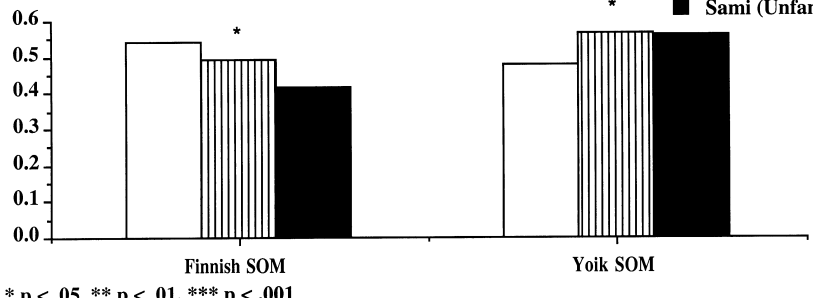

Fig. 11. The left shows the correlations between the Finnish SOM and the fitness judgments made by Western, Finnish, and Sami listeners. The right shows the correlations between the Yoik SOM and the fitness judgments made by Western, Finnish, and Sami listeners. The top graphs show the results for all yoiks, the second graph shows the results for the yoiks known only to the Sami listeners, the third graph shows the results for the yoiks known only to the Finnish listeners, and the bottom graph shows the results for the yoiks not known to any of the groups of listeners.

the results for the yoiks unfamiliar to all subjects. As can be seen in the graphs on the left, there was a trend for the highest correlations of the Finnish SOM to be with the Western data, followed by the Finnish data, and lowest for the Sami data. When individual subject correlations were compared, this difference was found to be significant only in the lowest graph; the corresponding $F$ values for differences between groups were $F(2,36)=1.84,0.58,0.27$, and 3.33 , respectively, all of which are non-significant except for the last, with $P<0.05$. It may appear puzzling 
that the correlations between the Finnish SOM and Western subjects should be somewhat higher than with the Finnish subjects. It should be noted that in no case was this difference significant. Recall also that the songs used to train the Finnish SOM would be stylistically familiar to the Western subjects, and that the Finnish listeners were responding to yoik contexts from a style that was somewhat familiar to them.

The graphs on the right of Fig. 11 show the correlations between the fitness measures of the Yoik SOM and the average behavioral data from all three groups of subjects. In all cases, the differences between groups were significant, with the strongest difference found in the second graph for the yoiks familiar to the Sami subjects but not the others. The corresponding $F$ values for differences between groups were $F(2,36)=5.22, \quad P<0.01, \quad F(2,36)=6.44, \quad P<0.005$, $F(2,36)=4.91, P<0.02$, and $F(2,36)=4.06, P<0.03$, for the four graphs, respectively. Thus, as expected, on average the Yoik SOM modeled the behavioral responses of the Sami subjects better than the other groups, and this was especially so for the yoiks familiar to the Sami subjects.

\subsubsection{Bottom-up principles: the implication-realization model and consonance}

The fitness measures of the Yoik SOM were analyzed in terms of the implicationrealization model's principles and consonance (using Model 2 of Table 5). Recall that this model coded Proximity and Registral Return as linear distance from the last tone of the context and the second to last tone of the context, respectively. The multiple correlation for Model 2 and the Yoik SOM was highly significant $\left(R(7,168)=0.47, P<0.0001\right.$, adjusted $\left.R^{2}=0.23\right)$. Fig. 12 shows the simple correlations between the seven variables of Model 2 and the Yoik SOM. As with the behavioral data for the Sami subjects (as well as the Finnish subjects, compare Fig. 8), the two consonance variables were strong and highly significant. For the Yoik SOM, none of the other variables of the model was significant, either in the simple or multiple correlations.

The fitness measures of the Finnish SOM were also analyzed in terms of this model. The multiple correlation for Model 2 and the Finnish SOM was again highly significant $\left(R(7,168)=0.58, P<0.0001\right.$, adjusted $\left.R^{2}=0.34\right)$. Fig. 12 also shows the simple correlations between the seven variables of Model 2 and the Finnish SOM. As with the behavioral data for the Western subjects, the correlations with Intervallic Difference, Registral Return (linear), and Proximity (linear) were relatively strong and significant. The two Consonance variables were also relatively strong. All these variables were significant in both the multiple and simple correlations except for Proximity, which was only marginally significant in the multiple correlation.

Compared to the behavioral data (Table 5), the multiple correlations for the implication-realization model were lower for the fitness measures of the two SOMs. Some aspects of melodic processing in humans are apparently not captured by the SOM model. However, the multiple correlations for the SOM fitness measures were still highly significant, suggesting that the SOM model abstracted to some degree the underlying principles coded in the implication-realization model. 


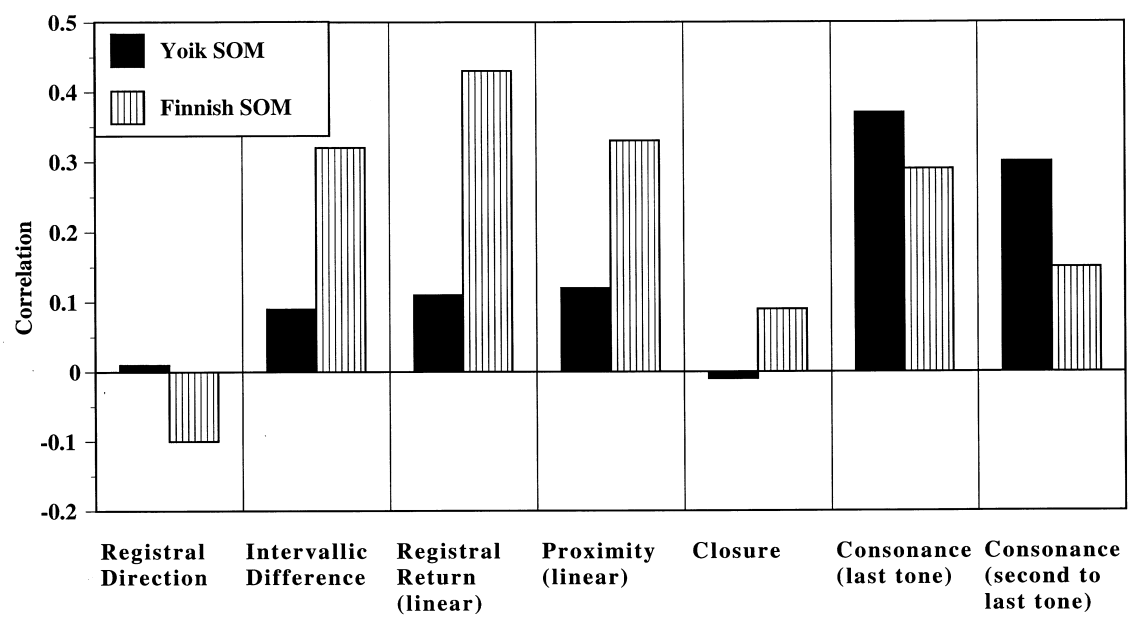

Fig. 12. The simple correlations between the fitness measures of the Yoik SOM and the Finnish SOM and the factors in Model 2. These include principles of the Narmour (1990) implication-realization model's principles (as modified by Krumhansl, 1995a) and consonance.

Moreover, the relative weights underlying the Yoik SOM were similar to those underlying the behavioral data for the Sami subjects (and the Finnish subjects), and the relative weights underlying the Finnish SOM were similar to those underlying the behavioral data of the Western subjects. Thus, contrasts between the models in terms of the weights of the model's principles corresponded with differences between the groups of listeners.

\subsubsection{Western schematic versus veridical expectations}

The two-tone context data (Krumhansl, 1995a) are again taken as representative of Western schematic knowledge. The correlations between these data and the fitness measures for the two SOMs are shown at the left of Fig. 13. As can be seen, the correspondence with schematic Western knowledge was greater for the Finnish SOM than the Yoik SOM. For the veridical variable which codes the correct next tone, the inverse pattern was found, as seen in the right of Fig. 13. In sum, the Finnish SOM abstracted information from its corpus that produced fitness measures mirroring previous behavioral judgments given by Western subjects to simple two-tone contexts (Krumhansl, 1995a). In contrast, the Yoik SOM's fitness judgments quite strongly reflected the correct next tone in the yoiks. These results parallel those found for the different groups of subjects in the behavioral experiment (compare Fig. 9).

\section{Convergence between the three approaches}

Table 6 shows the correlations between the results of the three approaches. The top of the table shows the results of the statistical style analysis (going from tone distributions, to two- and three-tone transitions, to the correct next tone) compared 


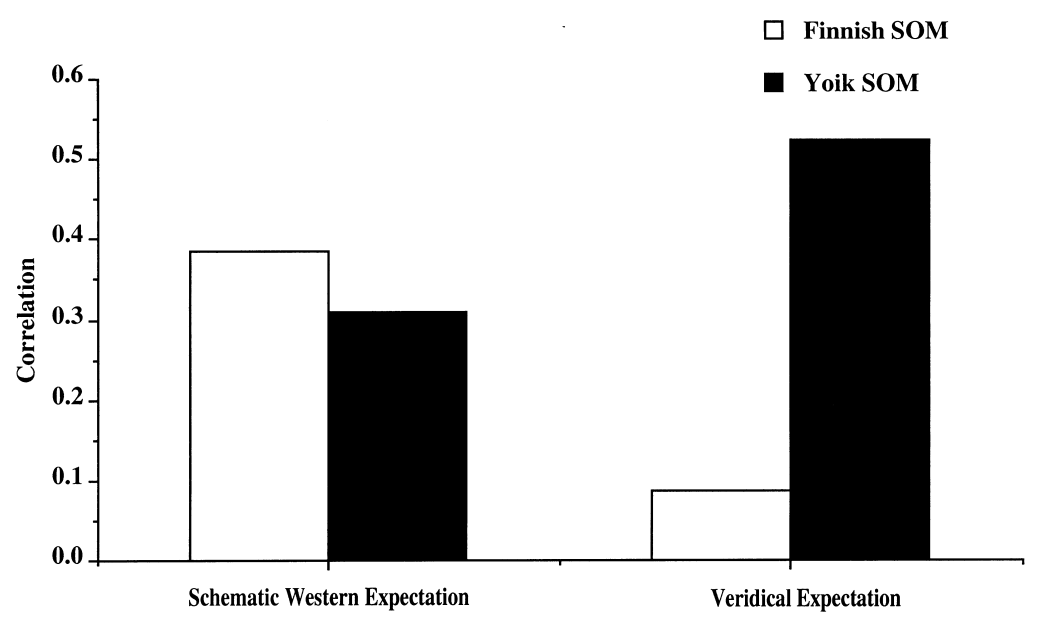

Fig. 13. The left shows the correlations between schematic Western expectation (as measured by Krumhansl, 1995a) and the Yoik and Finnish SOMs. The right shows the correlations between veridical expectation (correct next tone), and the Yoik and Finnish SOMs.

with the behavioral experiment and the SOMs. As can be seen, the correlations are all highly significant, with the exception of the correlation between the correct next tone and either the Western subjects or the Finnish SOM. These exceptions would be expected because none of the Western subjects were familiar with the yoiks, and the Finnish SOM was not trained with the yoiks. At a general level, these correlations with the statistical style analysis are important for two reasons. First, they reinforce previous experimental results that suggested that listeners are sensitive to statistical distributions of tones (e.g. Castellano et al., 1984; Krumhansl, 1990; Oram \& Cuddy, 1995). The present results show that they are sensitive not only to the tone distributions, but also the higher-order statistics, the two- and three-tone transitions. This finding was also obtained in the study by Krumhansl et al. (1999) of Finnish spiritual folk hymns. Second, the correlations between the statistical analysis and the SOM show that the SOM model has also abstracted the same statistical properties of music. This reinforces the view that the SOM models human learning, in as much as both appear to encode frequently occurring tones and tone combinations.

The remainder of Table 6 shows for completeness the correlations between the behavioral data and the SOM model. As discussed above, the correlations between groups (shown on the bottom left) suggested that the Finnish group is intermediate between the Western and Sami group, which had the lowest correlation with one another. These differences were relatively small, however. Overall, the correlations between groups, who differ considerably in their experience with the Sami yoik tradition, were quite strong. This can be understood in terms of two kinds of psychological principles. The first, just discussed, is that all groups appear sensitive to the distribution of tones and tone sequences. The second, discussed in connection 
Table 6

Convergence between approaches

\begin{tabular}{|c|c|c|c|c|c|}
\hline & \multicolumn{3}{|c|}{ Behavioral experiment } & \multicolumn{2}{|c|}{ SOM model } \\
\hline & Western & Finnish & Sami & Finnish & Sami \\
\hline \multicolumn{6}{|l|}{ Statistical style analysis } \\
\hline Tone distributions & $0.63 * * * a$ & $0.74 * * *$ & $0.66 * * *$ & $0.48 * * *$ & $0.62 * * *$ \\
\hline Two-tone distributions & $0.67 * * *$ & $0.61 * * *$ & $0.57 * * *$ & $0.53 * * *$ & $0.48 * * *$ \\
\hline Three-tone distributions & $0.41 * * *$ & $0.38 * * *$ & $0.43 * * *$ & $0.40 * * *$ & $0.52 * * *$ \\
\hline Correct next tone & 0.11 & $0.21 * *$ & $0.32 * * *$ & 0.09 & $0.52 * * *$ \\
\hline \multicolumn{6}{|l|}{ Behavioral experiment } \\
\hline Western & - & $0.91 * * *$ & $0.82 * * *$ & $0.59 * * *$ & $0.53 * * *$ \\
\hline Finnish & $0.91 * * *$ & - & $0.87 * * *$ & $0.52 * * *$ & $0.62 * * *$ \\
\hline Sami & $0.82 * * *$ & $0.87 * * *$ & - & $0.46^{* * *} *$ & $0.63 * * *$ \\
\hline
\end{tabular}

a $* * P<0.01, * * * P<0.0001$

with the implication-realization model (Narmour, 1990), is the finding that similar bottom-up principles (notably consonance) influenced the responses of all listeners.

The bottom right of the figure shows the correlations between the behavioral results and the Finnish and Yoik SOM models. The same two kinds of principles can be used to explain the convergence between the behavioral judgments and the SOM fitness measures. First, both listeners and SOMs correlated with the statistical distributions of tones and tone sequences. Second, the SOM's weights of the principles of the implication-realization model and consonance resembled those of the listeners. Looking in more detail at the pattern of correlations, we see that the Finnish SOM correlated more highly with the judgments of Western listeners, whereas the Sami SOM correlated more strongly with the Finnish and Sami listeners. This can be accounted for by the finding that the Sami SOM's weights on the implication-realization model's principles and consonance were similar to those for the Finnish and Sami listeners. In contrast, the weights for the Finnish SOM more strongly resembled those of the Western listeners. In addition, the Sami and, to some extent, the Finnish groups showed relatively strong veridical expectations for the yoiks with which they were familiar, and the Yoik SOM also evidenced strong veridical expectations. In contrast, the Western schematic expectations were stronger for the Western subject and the Finnish SOM. Thus, decomposing the results into underlying influences on melodic expectations enabled us to account for differences between the SOMs as they relate to the behavioral data.

\section{Conclusions}

We believe that a combination of methods, such as those used here, is important for establishing the convergence between different approaches applied to a single topic. The topic of this study, musical expectancy, was chosen because previous 
research allowed us to compare the present behavioral results with those for other styles of music and more schematic materials. Theoretical proposals about melodic expectancy provided testable predictions about underlying psychological principles. The statistical style analysis was useful for finding both general stylistic features and more specific characteristics of the style, and determining their influence on the behavioral judgments. Finally, the SOM model was tested as to whether it is an adequate model of learning melodic structure. Comparing it with the behavioral and statistical style measures examined whether it follows the same psychological principles and is sensitive to the same musical features. This strategy, used in a crosscultural context, has the additional advantage that it can uncover effects of musical expertise within a musical culture and distinctive properties of musical styles.

We close with some generalizations and some important caveats. Perhaps the most significant finding of the behavioral experiment was that listeners appear to adapt relatively easily to different ways of structuring sound in music. All three groups produced generally similar responses with one another. This finding is analogous to previous studies showing strong agreement between listeners from different musical cultures (Castellano et al., 1984; Krumhansl, 1995b). In the present study, there were some residual effects of expertise which depended as much on the listeners' familiarity with the particular yoiks and the listeners' musical culture. The Sami listeners' judgments of melodic continuations were more strongly influenced by knowledge of the yoiks than the Finnish listeners, but only when the yoiks were highly familiar to the Sami group. For the yoiks that were familiar to the Finnish listeners and unfamiliar to the Sami listeners, the responses of the two groups were very similar. Thus, experience with the particular yoiks can compensate for lack of style knowledge. Finally, as in the earlier studies, non-expert listeners were apparently largely able to suppress their knowledge of Western music. All three groups of subjects produced responses that were relatively dissimilar from schematic Western expectations (Krumhansl, 1995a). However, the finding that schematic Western expectations had a greater influence on the Western listeners than the other two groups suggested that their adjustment was incomplete. Results such as these, analyzed in terms of different aspects of the listeners experience, promise insights into the types of experience that lead to musical expertise.

In addition, similar underlying principles were found for the Sami and the Finnish groups. Western listeners diverged somewhat from the other two groups, again indicating residual influences of Western tonal music. In particular, we used the bottom-up principles of the Narmour (1990) implication-realization model together with consonance, known to be perceptually relevant. Even though the relative weighting of these principles was different from Western music, the principles had similar effects on the Sami and Finnish listeners. The Western group's weighting resembled the other groups, with some apparent additional influence of Western tonal music. Comparison with the results of the same analysis for Finnish spiritual folk hymns (Krumhansl et al., 1999) suggests that musical styles may share a core of basic principles, but that their relative importance varies across styles. The most notable difference between these studies was the shift in importance from proximity in the folk hymns to consonance in the yoiks. All listeners in the present experiment 
appeared sensitive to other distinctive features of the yoiks, such as the presence of large intervals, the pentatonic orientation of the yoiks with possibly two central reference pitches, and the greater variability of melodic continuations allowed within the yoik tradition.

The statistical style analysis of tones in a corpus of yoiks was able to characterize more precisely the properties of the yoiks, including the pentatonic orientation, the presence of two reference pitches, the frequency of large consonant intervals, and the relatively unconstrained set of continuation tones. Moreover, the convergence of the statistical results with the behavioral measures reinforced the idea that listeners learn a musical style in part by attending to statistical regularities. The strong agreement between groups can be explained by the idea that even listeners who are inexperienced with the style can appreciate some aspects of its organization by attending to statistical properties of the music, such as the number of times that different tones and tone-combinations appear in the presented musical contexts (see also Castellano et al., 1984; Kessler et al., 1984; Krumhansl, 1990; Krumhansl et al., 1999; Oram \& Cuddy, 1995).

The SOM model matched the behavioral responses to a fair degree despite its restricted training corpus and comparative computational simplicity. More important than this general agreement was the finding that the SOM model qualitatively mirrored detailed aspects of the behavioral responses. First, the SOM demonstrated sensitivity to the statistics of tone distributions and tone transitions as did the listeners. Second, the SOM modeled differences between the groups of listeners. Two different SOMs, one trained on a corpus of Lutheran hymns and Finnish folk songs, the other on the experimental sequences including the correct next tone, were able to characterize effects of expertise. Third, the SOMs and the behavioral responses were both influenced by similar underlying melodic principles including some of those proposed in the Narmour (1990) implication-realization model. Finally, the weighting of the principles differed across listener groups in the same way that they differed across the SOMs trained with the different musical corpora.

That the SOM model reflected the Narmour (1990) implication-realization model's principles to some degree raises the possibility that the principles might be abstracted from listening to music. As shown by Thompson and Stainton (1998), music at least in one style tends to conform to the principle of the implicationrealization model (Narmour, 1990). If this is generally true, then it is possible that listeners have abstracted the principles because they describe common patterns in the music they have heard. With the SOM approach, the music presented can be precisely controlled, suggesting that this kind of modeling offers a special opportunity for studying the psychological origin of the proposed bottom-up principles. These findings suggest that the SOM model might also play a useful role in future ethnomusicological studies. The SOM model has two advantages in this context. First, its input is acoustic information and so it does not require musical transcription which is subject to cultural bias. Second, it does not entail the kinds of difficulties involved in observational or behavioral studies in the field. Thus, it might serve a useful function in generating hypotheses for further examination with other methods. 
These findings should be viewed in light of the study's limitations, however. First, and most obviously, we investigated only one aspect of perceptual responses, expectancy for melodic continuations. Moreover, we did so with an artificial task in which the melody was interrupted and different possible continuation tones were added. Despite the artificiality, all listeners adjusted easily to the task demands. However, it would be desirable to examine melodic expectancy in a more musically natural setting. The listeners were apparently not disturbed by the synthesized sound required for the study, but additional influences of vocal timbre can not be ruled out. In addition, although the present study was able to separate effects of style knowledge from effects of familiarity, more systematic study of this question is needed. This would necessitate advanced information about the melodies familiar to the target listener groups. Lastly, we have considered here only one style of music, Sami yoiks, although we were able to compare the results with those using the same methods with a quite different style, Finnish spiritual folk hymns. This comparison only begins to explore the richness and variety found across musical cultures.

Finally, the generality of findings from studies such as this may be limited by the rapid transitions that are occurring in the ways different musical cultures are influencing one another. This suggests some urgency in conducting cross-cultural studies. We believe that it is important for the general assumptions of the relevant disciplines to be tested with a broad range of methods. The present findings, using cognitive methodologies, challenge the common assumption of ethnomusicology that extensive experience of music within its cultural context is essential for its basic structural principles to be apprehended. This does not mean that more detailed, culturally-based methodologies should not be applied in addition. Field work studies are needed that aim to understand the subjective significance of music in its cultural context and other issues that can be addressed well with qualitative data based on interviews and observations. These studies are important for developing new theories, models, and hypotheses that might then be tested in the future with other methods and within other theoretical frameworks.

\section{Acknowledgements}

We thank Ilpo Saastamoinen for consultation on the project, and especially the expert yoikers who participated in the experiment. We are greatly indebted to Annukka Hirvasvuopio from Utsjoki, Finland, for extensive contributions to this research, including serving as our first expert subject, translating the instructions into Sami language, arranging for the field work in Kautokeino, Norway, traveling with us there, and contacting the subjects and supervising the experimental sessions. Her generous contributions and insightful suggestions are gratefully acknowledged. Finally, we are thankful to two anonymous reviewers for constructive suggestions on a previous version of this article. The research was supported by a Fulbright fellowship, 'Music cognition: Cultural and neural substrates', and a faculty grant from Cornell University to C.L.K. It was also supported by the Finnish Academy with the grant 'Tacit knowledge in complex mind environment systems' and a grant 
from the University of Jyväskylä. T.J. was supported by a post-doctoral research grant from the Finnish Academy, and T.E. was supported by a pre-doctoral research grant from the University of Jyväskylä.

\section{Appendix A}

Table 7 shows all of the three-tone transitions for the corpus of 18 yoiks used in the statistical style analysis of tones. Each line in each column has the frequency followed by the tones in the three-tone transition. The transitions that were used in the analysis of the behavioral data are marked with an asterisk (*).

\section{Appendix B}

Fig. 14 shows the linear coding of Proximity and Registral Return (Krumhansl, 1995a), and the original quantitative coding of the principles of Registral Direction, Intervallic Difference, and Closure in Narmour's implication-realization model (Krumhansl, 1995b). Table 8 shows the consonance values used. These are the average of the normalized values of the empirical and theoretical values of consonance summarized in Krumhansl (1990, p. 57).

\section{Appendix C. Discography}

\section{C.1. Traditional}

Angel Nieiddat/Angelin tytöt (1992). Dolla (MIPUCD 102), Mipu Music, Finland.

Matts Arnberg, Hakan Unsggard, Israel Ruong (1997). Jojk - A Presentation of Sami Folk Music (CAP 21544 300), Caprice Record, Sweden.

Johan Anders Baer (1991). Máhkarávju (DATCD 12), Guovdageaidnu, Norway. Anders P. Bongo (1999). Nássa (DATCD 28), Guovdageaidnu, Norway.

Mattis Haetta (1980). Juoiga (Jorgaleaddji JLP-1006).

Mattis Haetta (1983). Maze (Jorgaleaddji JMC-1007; available only as a cassette).

Mattis Haetta (1980) Låla; a collaboration with Norwegian singer Sverre Kjellsberg (Folklore MAI-8009).

Inga Juuso (1991). Ravddas Ravdii (DAT CD-9), Guovdageaidnu, Norway.

Rávnna-Jon Rávna and Pieras-Per-Anne Ristin (1995). Calbmelan (DATCD-22), Guovdageaidnu, Norway.

Nils-Aslak Valkeapää (1998). The Magic of Sámi Yoik (Finlandia Innovators 3984-22112-2).

Wimme (1995). Wimme (ZENCD 2043, in G-A-S: EXIL 5522), Rockadillo Records, Finland. USA release: Wimme (1997). Wimme (Northside, NSD 6005), USA. 
Table 7

Three-tone transitions in yoiks

\begin{tabular}{|c|c|c|c|c|c|c|c|c|c|c|c|c|c|c|c|c|c|c|c|}
\hline 49 & $\mathrm{~d}$ & $\mathrm{~d}$ & $\mathrm{c}$ & 12 & $\mathrm{~d}$ & $\mathrm{c}$ & $\mathrm{g}^{*}$ & 4 & $\mathrm{c}$ & $\mathrm{c}$ & $\mathrm{g}$ & 2 & $\mathrm{c}$ & A & $\mathrm{G}$ & 2 & $\mathrm{a}$ & $\mathrm{c}^{\prime}$ & $\mathrm{c}^{\prime}$ \\
\hline 39 & $\mathrm{e}$ & $\mathrm{d}$ & $\mathrm{c}^{*}$ & 12 & $\mathrm{e}$ & $\mathrm{G}$ & $\mathrm{e}$ & 4 & $\mathrm{c}$ & $\mathrm{c}$ & $\mathrm{a}$ & 2 & $\mathrm{c}$ & $\mathrm{d}$ & $a^{*}$ & 2 & $\mathrm{c}^{\prime}$ & $\mathrm{c}$ & $\mathrm{d}$ \\
\hline 36 & $\mathrm{c}$ & $\mathrm{c}$ & $\mathrm{G}$ & 12 & $\mathrm{~g}$ & $\mathrm{e}$ & $\mathrm{c}$ & 4 & $\mathrm{c}$ & $\mathrm{d}$ & $\mathrm{G}^{*}$ & 2 & $\mathrm{c}$ & $\mathrm{g}$ & $\mathrm{d}$ & 2 & $\mathrm{c}^{\prime}$ & $\mathrm{g}$ & $\mathrm{G}$ \\
\hline 36 & $\mathrm{~d}$ & $\mathrm{c}$ & $\mathrm{c}^{*}$ & 12 & $\mathrm{~g}$ & $\mathrm{~g}$ & $\mathrm{e}$ & 4 & $\mathrm{c}$ & $\mathrm{g}$ & $\mathrm{a}$ & 2 & $\mathrm{c}$ & $\mathrm{g}$ & $\mathrm{e}$ & 2 & $\mathrm{c}^{\prime}$ & $\mathrm{g}$ & $\mathrm{d}$ \\
\hline 35 & $\mathrm{c}$ & $\mathrm{d}$ & $\mathrm{d}^{*}$ & 12 & $\mathrm{~g}$ & $\mathrm{~g}$ & $\mathrm{a}$ & 4 & $\mathrm{c}$ & $\mathrm{a}$ & $\mathrm{g}$ & 2 & $\mathrm{c}$ & $\mathrm{g}$ & $\mathrm{f}$ & 2 & $\mathrm{c}^{\prime}$ & $\mathrm{a}$ & c \\
\hline 35 & $\mathrm{c}$ & $\mathrm{e}$ & $\mathrm{g}$ & 12 & $\mathrm{a}$ & $\mathrm{a}$ & $\mathrm{g}$ & 4 & $\mathrm{c}$ & $\mathrm{c}^{\prime}$ & $\mathrm{d}^{\prime}$ & 2 & $\mathrm{c}$ & $\mathrm{c}^{\prime}$ & $\mathrm{g}$ & 2 & $\mathrm{c}^{\prime}$ & $\mathrm{a}$ & $\mathrm{g}$ \\
\hline 34 & $\mathrm{e}$ & $\mathrm{e}$ & d & 11 & $\mathrm{~d}$ & $\mathrm{e}$ & d & 4 & $\mathrm{~d}$ & $\mathrm{~g}$ & $\mathrm{a}$ & 2 & $\mathrm{c}$ & $\mathrm{d}^{\prime}$ & $\mathrm{c}^{\prime}$ & 2 & $\mathrm{c}^{\prime}$ & $\mathrm{a}$ & $\mathrm{a}$ \\
\hline 33 & $\mathrm{G}$ & $\mathrm{c}$ & $\mathrm{c}$ & 11 & $\mathrm{e}$ & $\mathrm{c}$ & $\mathrm{d}$ & 4 & $\mathrm{e}$ & $\mathrm{g}$ & $\mathrm{e}$ & 2 & $\mathrm{c}$ & $\mathrm{d}^{\prime}$ & $\mathrm{e}^{\prime}$ & 2 & $\mathrm{c}^{\prime}$ & $\mathrm{c}^{\prime}$ & c \\
\hline 33 & $\mathrm{c}$ & $\mathrm{c}$ & $\mathrm{d}$ & 10 & c & $\mathrm{e}$ & $\mathrm{c}$ & 4 & $\mathrm{f}$ & $\mathrm{G}$ & $\mathrm{d}$ & 2 & $\mathrm{~d}$ & $\mathrm{G}$ & $\mathrm{e}$ & 2 & $\mathrm{c}^{\prime}$ & $\mathrm{c}^{\prime}$ & $\mathrm{g}$ \\
\hline 32 & $\mathrm{~d}$ & $\mathrm{e}$ & $\mathrm{G}$ & 10 & $\mathrm{~d}$ & $\mathrm{~d}$ & $\mathrm{~g}$ & 4 & $\mathrm{~g}$ & $\mathrm{G}$ & $\mathrm{e}$ & 2 & $\mathrm{~d}$ & $\mathrm{G}$ & $\mathrm{g}$ & 2 & $\mathrm{c}^{\prime}$ & $\mathrm{c}^{\prime}$ & $\mathrm{a}$ \\
\hline 31 & $\mathrm{c}$ & $\mathrm{d}$ & $\mathrm{e}^{*}$ & 10 & $\mathrm{~d}$ & $\mathrm{~g}$ & $\mathrm{e}$ & 4 & $\mathrm{~g}$ & $\mathrm{f}$ & $\mathrm{f}$ & 2 & $\mathrm{~d}$ & A & $\mathrm{G}$ & 2 & $\mathrm{c}^{\prime}$ & $\mathrm{d}^{\prime}$ & $\mathrm{a}$ \\
\hline 28 & $\mathrm{~d}$ & $\mathrm{c}$ & $\mathrm{d}^{*}$ & 9 & $\mathrm{G}$ & $\mathrm{d}$ & $\mathrm{e}$ & 4 & $\mathrm{~g}$ & $\mathrm{f}$ & $\mathrm{G}$ & 2 & $\mathrm{~d}$ & B & $\mathrm{G}$ & 2 & $c^{\prime}$ & $\mathrm{d}^{\prime}$ & $\mathrm{c}^{\prime}$ \\
\hline 27 & $\mathrm{c}$ & $\mathrm{G}$ & $\mathrm{c}^{*}$ & 8 & $\mathrm{G}$ & $\mathrm{G}$ & $\mathrm{c}^{*}$ & 4 & $\mathrm{~g}$ & $\mathrm{c}^{\prime}$ & $\mathrm{a}$ & 2 & $\mathrm{~d}$ & c & $\mathrm{d}^{\prime *}$ & 2 & $\mathrm{c}^{\prime}$ & $\mathrm{e}^{\prime}$ & $\mathrm{e}^{\prime}$ \\
\hline 25 & $\mathrm{c}$ & $\mathrm{G}$ & $\mathrm{e}^{*}$ & 8 & $\mathrm{G}$ & A & $\mathrm{G}$ & 4 & $\mathrm{~g}$ & $\mathrm{c}^{\prime}$ & $\mathrm{c}^{\prime}$ & 2 & $\mathrm{~d}$ & $\mathrm{~d}$ & A & 2 & $\mathrm{~d}^{\prime}$ & $\mathrm{d}$ & $\mathrm{d}$ \\
\hline 25 & $\mathrm{e}$ & $\mathrm{G}$ & $\mathrm{c}$ & 8 & $\mathrm{e}$ & $\mathrm{d}$ & $\mathrm{G}^{*}$ & 4 & $\mathrm{a}$ & $\mathrm{g}$ & $\mathrm{f}$ & 2 & $\mathrm{~d}$ & $d$ & B & 2 & $\mathrm{~d}^{\prime}$ & $\mathrm{a}$ & $\mathrm{g}$ \\
\hline 25 & $\mathrm{e}$ & $\mathrm{g}$ & $\mathrm{g}$ & 8 & $\mathrm{~g}$ & $\mathrm{a}$ & $\mathrm{e}$ & 4 & $\mathrm{c}^{\prime}$ & $\mathrm{d}$ & $\mathrm{d}$ & 2 & $\mathrm{~d}$ & $\mathrm{e}$ & A & 2 & $\mathrm{~d}^{\prime}$ & $\mathrm{c}^{\prime}$ & $\mathrm{c}^{\prime *}$ \\
\hline 24 & $\mathrm{c}$ & $\mathrm{c}$ & $\mathrm{e}$ & 8 & $\mathrm{a}$ & $\mathrm{e}$ & $\mathrm{c}$ & 4 & $\mathrm{c}^{\prime}$ & $\mathrm{c}^{\prime}$ & $\mathrm{d}$ & 2 & $\mathrm{~d}$ & $\mathrm{~g}$ & $\mathrm{f}$ & 2 & $\mathrm{~d}^{\prime}$ & $\mathrm{c}^{\prime}$ & $\mathrm{e}^{\prime *}$ \\
\hline 24 & $\mathrm{~d}$ & $\mathrm{c}$ & $\mathrm{e}^{*}$ & 8 & $\mathrm{a}$ & g & $\mathrm{e}$ & 4 & $\mathrm{c}^{\prime}$ & $\mathrm{c}^{\prime}$ & $\mathrm{c}^{\prime}$ & 2 & $\mathrm{~d}$ & $\mathrm{~g}$ & $\mathrm{~g}$ & 2 & $\mathrm{~d}^{\prime}$ & $\mathrm{d}^{\prime}$ & $\mathrm{d}$ \\
\hline 24 & $\mathrm{e}$ & $\mathrm{c}$ & $\mathrm{c}$ & 7 & $\mathrm{G}$ & $\mathrm{G}$ & $\mathrm{g}^{*}$ & 3 & $\mathrm{G}$ & $\mathrm{c}$ & $\mathrm{e}$ & 2 & $\mathrm{~d}$ & $\mathrm{a}$ & $\mathrm{a}$ & 2 & $\mathrm{~d}^{\prime}$ & $\mathrm{e}^{\prime}$ & $\mathrm{a}$ \\
\hline 21 & $\mathrm{~g}$ & $\mathrm{~d}$ & $\mathrm{~d}$ & 7 & $\mathrm{G}$ & $\mathrm{e}$ & $\mathrm{G}$ & 3 & $\mathrm{G}$ & $\mathrm{g}$ & $\mathrm{a}$ & 2 & e & $\mathrm{G}$ & $\mathrm{G}$ & 2 & $\mathrm{e}^{\prime}$ & $\mathrm{a}$ & $\mathrm{g}$ \\
\hline 21 & $\mathrm{~g}$ & $\mathrm{e}$ & $\mathrm{d}$ & 7 & $\mathrm{G}$ & $\mathrm{e}$ & $\mathrm{c}$ & 3 & $\mathrm{c}$ & $\mathrm{g}$ & $\mathrm{c}^{\prime}$ & 2 & $\mathrm{e}$ & $\mathrm{G}$ & A & 2 & $\mathrm{e}^{\prime}$ & $\mathrm{d}^{\prime}$ & $\mathrm{d}^{\prime}$ \\
\hline 19 & c & $\mathrm{d}$ & $\mathrm{c}^{*}$ & 7 & $\mathrm{c}$ & $\mathrm{e}$ & $\mathrm{e}$ & 3 & $\mathrm{e}$ & $\mathrm{f}$ & $\mathrm{e}$ & 2 & $\mathrm{e}$ & A & A & 2 & $\mathrm{e}^{\prime}$ & $\mathrm{e}^{\prime}$ & $\mathrm{d}^{\prime}$ \\
\hline 19 & $\mathrm{e}$ & $\mathrm{d}$ & $\mathrm{e}^{*}$ & 7 & $\mathrm{~g}$ & $\mathrm{G}$ & $\mathrm{G}$ & 3 & $\mathrm{e}$ & $\mathrm{g}$ & $\mathrm{f}$ & 2 & $\mathrm{e}$ & $\mathrm{d}$ & $g^{*}$ & 1 & $\mathrm{G}$ & $\mathrm{d}$ & $\mathrm{G}$ \\
\hline 18 & $\mathrm{G}$ & $\mathrm{e}$ & $\mathrm{e}$ & 7 & d & $\mathrm{G}$ & $\mathrm{c}$ & 3 & $\mathrm{f}$ & $\mathrm{e}$ & $\mathrm{d}$ & 2 & e & e & $\mathrm{G}$ & 1 & $\mathrm{G}$ & $\mathrm{f}$ & f \\
\hline 18 & $\mathrm{e}$ & $\mathrm{c}$ & $\mathrm{G}$ & 6 & $\mathrm{G}$ & $\mathrm{G}$ & $\mathrm{e}^{*}$ & 3 & $\mathrm{f}$ & $\mathrm{e}$ & $\mathrm{g}$ & 2 & $\mathrm{f}$ & $\mathrm{G}$ & f & 1 & $\mathrm{G}$ & $\mathrm{g}$ & $\mathrm{e}$ \\
\hline 18 & $\mathrm{e}$ & $\mathrm{g}$ & $\mathrm{d}$ & 6 & $\mathrm{G}$ & $\mathrm{e}$ & $\mathrm{g}$ & 3 & $\mathrm{~g}$ & $\mathrm{G}$ & $\mathrm{d}$ & 2 & $\mathrm{f}$ & $\mathrm{c}$ & $\mathrm{d}^{\prime *}$ & 1 & c & $\mathrm{G}$ & $\mathrm{f}^{*}$ \\
\hline 16 & $\mathrm{c}$ & $\mathrm{c}$ & c & 6 & $\mathrm{G}$ & $\mathrm{a}$ & $\mathrm{g}$ & 3 & $\mathrm{~g}$ & $\mathrm{c}$ & $\mathrm{d}$ & 2 & $\mathrm{f}$ & $\mathrm{f}$ & $\mathrm{a}$ & 1 & $\mathrm{c}$ & $\mathrm{a}$ & $\mathrm{a}$ \\
\hline 16 & $\mathrm{~d}$ & $\mathrm{e}$ & $\mathrm{c}$ & 6 & $\mathrm{c}$ & $\mathrm{G}$ & $\mathrm{g}^{*}$ & 3 & $\mathrm{~g}$ & $\mathrm{~d}$ & $\mathrm{G}$ & 2 & $\mathrm{f}$ & $\mathrm{f}$ & $\mathrm{f}$ & 1 & $\mathrm{c}$ & b & $\mathrm{a}$ \\
\hline 16 & $\mathrm{~g}$ & $\mathrm{~d}$ & $\mathrm{c}$ & 6 & $\mathrm{c}$ & $\mathrm{G}$ & $a^{*}$ & 3 & $\mathrm{~g}$ & $\mathrm{e}$ & f & 2 & $\mathrm{f}$ & $\mathrm{f}$ & $\mathrm{G}$ & 1 & $\mathrm{~d}$ & $\mathrm{c}$ & $a^{*}$ \\
\hline 15 & $\mathrm{G}$ & $\mathrm{G}$ & $\mathrm{G}^{*}$ & 6 & $\mathrm{~d}$ & $\mathrm{G}$ & $\mathrm{G}$ & 3 & $\mathrm{~g}$ & $\mathrm{f}$ & $\mathrm{e}$ & 2 & $\mathrm{f}$ & $\mathrm{f}$ & $\mathrm{g}$ & 1 & $\mathrm{~d}$ & $\mathrm{c}$ & $\mathrm{b}^{*}$ \\
\hline 15 & $\mathrm{G}$ & $\mathrm{c}$ & $\mathrm{G}$ & 6 & $\mathrm{~d}$ & $\mathrm{G}$ & $\mathrm{d}$ & 3 & $\mathrm{~g}$ & $\mathrm{~g}$ & $\mathrm{f}$ & 2 & $\mathrm{f}$ & $\mathrm{g}$ & $\mathrm{a}$ & 1 & $\mathrm{~d}$ & $\mathrm{~d}$ & $\mathrm{~d}$ \\
\hline 15 & $\mathrm{~d}$ & $\mathrm{~d}$ & $\mathrm{e}$ & 6 & $\mathrm{~d}$ & $\mathrm{c}$ & $\mathrm{c}^{\prime} *$ & 3 & $\mathrm{a}$ & $\mathrm{g}$ & $\mathrm{c}$ & 2 & $\mathrm{f}$ & $\mathrm{g}$ & $\mathrm{c}$ & 1 & $\mathrm{e}$ & $\mathrm{G}$ & $\mathrm{d}$ \\
\hline 14 & $\mathrm{G}$ & $\mathrm{g}$ & $\mathrm{g}$ & 6 & $\mathrm{~g}$ & $\mathrm{c}$ & $\mathrm{c}$ & 2 & $\mathrm{G}$ & $\mathrm{G}$ & $\mathrm{a}^{*}$ & 2 & $\mathrm{f}$ & $\mathrm{a}$ & $\mathrm{g}$ & 1 & $\mathrm{f}$ & $\mathrm{g}$ & $\mathrm{g}$ \\
\hline 14 & $\mathrm{c}$ & $\mathrm{G}$ & $\mathrm{G}^{*}$ & 6 & $\mathrm{~g}$ & $\mathrm{~d}$ & g & 2 & $\mathrm{G}$ & $\mathrm{G}$ & $\mathrm{c}^{\prime *}$ & 2 & $\mathrm{~g}$ & $\mathrm{G}$ & A & 1 & $\mathrm{~g}$ & $\mathrm{e}$ & $\mathrm{e}$ \\
\hline 14 & $\mathrm{e}$ & $\mathrm{d}$ & $\mathrm{d}^{*}$ & 6 & $\mathrm{~g}$ & $\mathrm{~g}$ & c & 2 & $\mathrm{G}$ & $\mathrm{c}$ & A & 2 & $\mathrm{~g}$ & $\mathrm{G}$ & $\mathrm{g}$ & 1 & $\mathrm{~g}$ & $\mathrm{e}$ & $\mathrm{G}$ \\
\hline 14 & $\mathrm{~g}$ & $\mathrm{~g}$ & $\mathrm{G}$ & 6 & $\mathrm{a}$ & $\mathrm{g}$ & $\mathrm{d}$ & 2 & $\mathrm{G}$ & $\mathrm{c}$ & $\mathrm{g}$ & 2 & $\mathrm{~g}$ & $\mathrm{c}$ & $\mathrm{G}$ & 1 & $\mathrm{~g}$ & $\mathrm{f}$ & $\mathrm{g}$ \\
\hline 14 & $\mathrm{~g}$ & $\mathrm{~g}$ & $\mathrm{~d}$ & 5 & A & $\mathrm{G}$ & $\mathrm{g}$ & 2 & $\mathrm{G}$ & $\mathrm{f}$ & $\mathrm{g}$ & 2 & $\mathrm{~g}$ & $\mathrm{f}$ & $\mathrm{c}$ & 1 & $\mathrm{~g}$ & b & b \\
\hline 14 & $\mathrm{~g}$ & $\mathrm{~g}$ & $\mathrm{~g}$ & 5 & $\mathrm{c}$ & $\mathrm{g}$ & $\mathrm{g}$ & 2 & $\mathrm{G}$ & $\mathrm{a}$ & $\mathrm{a}$ & 2 & $\mathrm{~g}$ & $\mathrm{~g}$ & $\mathrm{c}^{\prime}$ & 1 & $\mathrm{a}$ & $\mathrm{g}$ & $\mathrm{b}$ \\
\hline 14 & $\mathrm{~g}$ & $\mathrm{a}$ & $\mathrm{g}$ & 5 & $\mathrm{~d}$ & $\mathrm{~d}$ & $\mathrm{G}$ & 2 & $\mathrm{G}$ & $\mathrm{c}^{\prime}$ & $\mathrm{c}^{\prime}$ & 2 & $\mathrm{~g}$ & $\mathrm{a}$ & $\mathrm{d}$ & 1 & $\mathrm{~b}$ & $\mathrm{c}$ & $\mathrm{d}$ \\
\hline 14 & $\mathrm{a}$ & $\mathrm{g}$ & $\mathrm{g}$ & 5 & $\mathrm{e}$ & $\mathrm{e}$ & $\mathrm{g}$ & 2 & A & $\mathrm{G}$ & $\mathrm{G}$ & 2 & $\mathrm{~g}$ & $\mathrm{a}$ & $\mathrm{c}^{\prime}$ & 1 & $\mathrm{~b}$ & $\mathrm{a}$ & $\mathrm{g}$ \\
\hline 13 & $\mathrm{~d}$ & $\mathrm{e}$ & $\mathrm{e}$ & 5 & $\mathrm{~g}$ & $\mathrm{a}$ & $\mathrm{a}$ & 2 & A & $\mathrm{G}$ & $\mathrm{d}$ & 2 & $\mathrm{a}$ & $c$ & $\mathrm{G}$ & 1 & $\mathrm{~b}$ & $\mathrm{~b}$ & $\mathrm{~b}$ \\
\hline 13 & $\mathrm{e}$ & $\mathrm{e}$ & e & 5 & $\mathrm{a}$ & $\mathrm{g}$ & $\mathrm{a}$ & 2 & A & $\mathrm{G}$ & e & 2 & $\mathrm{a}$ & $\mathrm{d}$ & $\mathrm{d}$ & 1 & $\mathrm{~b}$ & b & c \\
\hline 12 & $\mathrm{G}$ & $\mathrm{c}$ & $\mathrm{d}$ & 4 & $\mathrm{G}$ & $\mathrm{G}$ & $\mathrm{A}^{*}$ & 2 & A & $\mathrm{A}$ & $\mathrm{d}$ & 2 & $\mathrm{a}$ & g & $\mathrm{G}$ & & & & \\
\hline 12 & $\mathrm{G}$ & $\mathrm{e}$ & $\mathrm{d}$ & 4 & $\mathrm{G}$ & $\mathrm{d}$ & $\mathrm{d}$ & 2 & A & $\mathrm{d}$ & $\mathrm{G}$ & 2 & $\mathrm{a}$ & $\mathrm{g}$ & $\mathrm{c}^{\prime}$ & & & & \\
\hline 12 & $\mathrm{~d}$ & $\mathrm{c}$ & $\mathrm{G}^{*}$ & 4 & $\mathrm{G}$ & $\mathrm{g}$ & $\mathrm{d}$ & 2 & B & $\mathrm{G}$ & $\mathrm{G}$ & 2 & $\mathrm{a}$ & $\mathrm{a}$ & $\mathrm{a}$ & & & & \\
\hline
\end{tabular}




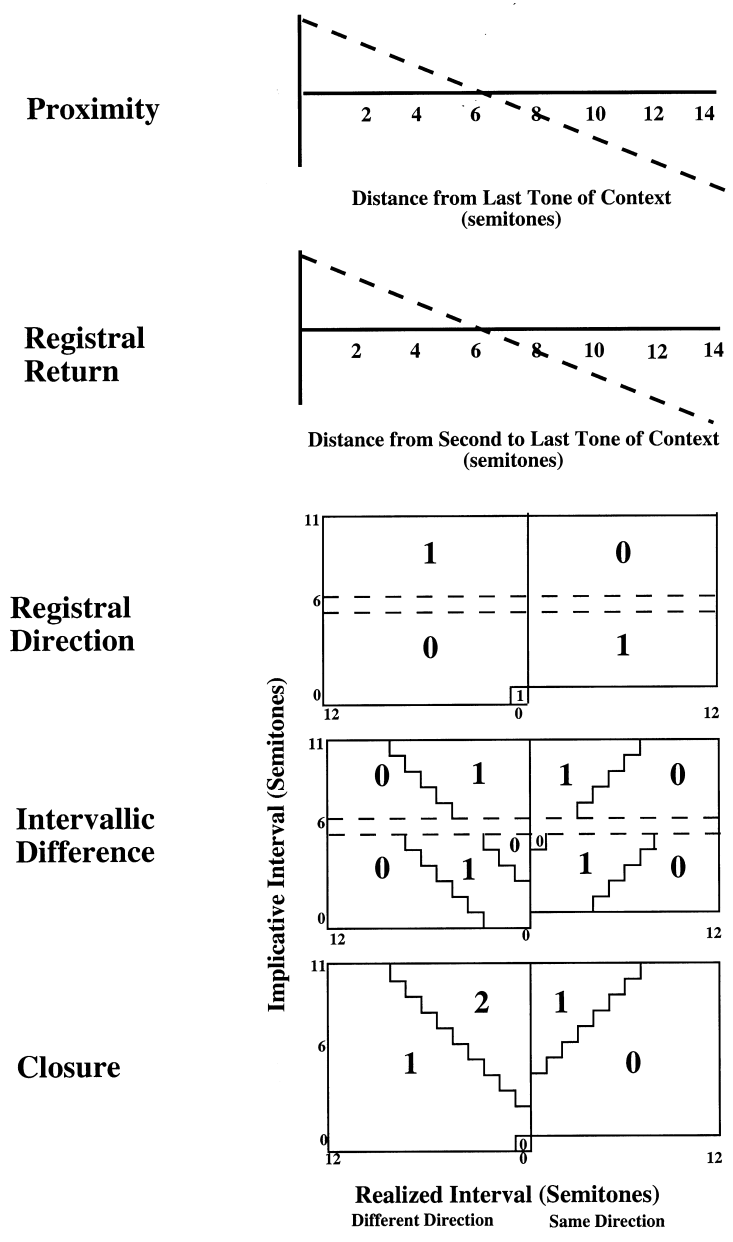

Fig. 14. The factors included in Model 2. Proximity and Registral Return are coded as linearly decreasing functions of pitch distance (as in Krumhansl, 1995a). Registral Direction, Intervallic Difference, and Closure are as originally coded (Krumhansl, 1995b). For these three principles, the horizontal axis represents the size of the realized interval in semitones and its direction (same or different) relative to the implicative interval.

\section{C.2. Compilations (traditional)}

Various (1992) Luodis Luohtái (ICD 921); includes yoiks by Elen Sara, Ole Gaino, Inga Tornensis and other yoikers from Norway.

Various (1997) 'Luodis Luohtái 2' (ICD 972): includes yoiks by Ole Larsen, Gaino, Mathis M. Gaup and others. 
Table 8

Consonance values used in Model 2

\begin{tabular}{lcr}
\hline Interval & Number of semitones & Consonance \\
\hline Unison & 0 & 1.466 \\
Minor second & 1 & -1.896 \\
Major second & 2 & -0.730 \\
Minor third & 3 & -0.031 \\
Major third & 4 & 0.428 \\
Perfect fourth & 5 & 0.719 \\
Tritone & 6 & -0.140 \\
Perfect fifth & 7 & 1.147 \\
Minor sixth & 8 & 0.079 \\
Major sixth & 9 & 0.620 \\
Minor seventh & 10 & -0.176 \\
Major seventh & 11 & -0.863 \\
\hline
\end{tabular}

\section{C.3. Contemporary, ambient, cross-over, etc.}

Angel Nieiddat/Angelin tytöt (1993). Giitu (Finlandia Innovators, 3984-268542), Finland.

Angel Nieiddat/Angelin tytöt (1995). Skeaikit (MIPUCD 402), Mipu Music, Finland.

Girls of Angeli (1997). The New Voice of North. Finlandia 0630-18063-2, Warner Music Finland.

Angelit (former Angelin Tytöt) (1999). Mánnu (Finlandia Innovators 398425790-2), Finland.

Mari Boine (1989). Gula Gula (ICD 891); Real World label and Virgin/EMI outside Scandinavia.

Mari Boine (1998). Room of Worship (Antilles 5590232).

Frode Fjellhem and Transjoik (1997). Mahkalahke (Atrium 0630 19780-2; also on ECM).

Ulla Pirttijärvi (1998). Ruossa Eanan (Atrium 0630 19717-2).

Wimme (1997). Gierran. Rockadillo Records ZENCD 2055, in G-A-S: EXIL 5536, Finland. USA release: Wimme (1999). Gierran. Northside, NSD 6023, USA.

\section{C.4. Recommended website}

http://www.digelius.com/sami.htm

\section{References}

Arnberg, M., Ruong, I., \& Unsgaard, H. (1969). Jojk/yoik. Stockholm: Swedish Radio Corporation, 1969/ 1998 (a pack of three CDs and a book).

Bakke, A. (1958). Innsamling av samisk folkemusikk (Collection of Sami folk music). Sameliv, 3, 17-21. Bharucha, J. J. (1987). Music cognition and perceptual facilitation: a connectionist framework. Music Perception, 5, 1-30. 
Bharucha, J. J., \& Todd, P. M. (1989). Modeling the perception of tonal structure with neural nets. Computer Music Journal, 13, 44-53.

Carlsen, J. C. (1981). Some factors which influence melodic expectancy. Psychomusicology, 1, 12-29.

Carlsen, J. C., Divenyi, P. L., \& Taylor, J. A. (1970). A preliminary study of perceptual expectancy in melodic configurations. Council for Research in Music Education Bulletin, 22, 4-12.

Castellano, M. A., Bharucha, J. J., \& Krumhansl, C. L. (1984). Tonal hierarchies in the music of North India. Journal of Experimental Psychology: General, 113, 394-412.

Cowan, N. (1988). Evolving conceptions of memory storage, selective attention, and their mutual constraints within the human information processing system. Psychological Bulletin, 104, 163-191.

Cowan, N. (1995). Attention and memory: an integrated framework. New York: Oxford University Press.

Cuddy, L. L., \& Lunney, C. A. (1995). Expectancies generated by melodic intervals: perceptual judgments of melodic continuity. Perception \& Psychophysics, 57, 451-462.

Danckert, W. (1956). Tonmalerei und Tonsymbolik in der Musik der Lappen (Tone color and tone symbol in the music of the Lapps). Die Musikforschung, 9, 286-296.

Darwin, C. J., Turvey, M. T., \& Crowder, R. G. (1972). An auditory analogue of the Sperling partial report procedure: evidence for brief auditory storage. Cognitive Psychology, 3, 255-267.

Edström, K. -O. (1978). [The Sami music culture]. Den samiska musikkulturen. Göteborg: Musikvetenskapliga Institutionen.

Ellingson, T. (1992). Transcription. In H. Myers, Ethnomusicology - an introduction (pp. 110-152). London: Macmillan.

Eriksson, J. (1986). Intervju med Ante Mikkel Gaup (Interview with Ante Mikkel Gaup). Kautokeino, Norway. Unpublished manuscript.

Fraisse, P. (1982). Rhythm and tempo. In D. Deutsch, The psychology of music. New York: Academic Press.

Freeman, L. C., \& Merriam, A. P. (1956). Statistical classification in anthropology: an application to ethnomusicology. American Anthropologist, 58, 464-472 Reprinted in K. K. Shelemay (1990). Garland library of readings in ethnomusicology. New York: Garland.

Huron, D. (1993). Chordal-tone doubling and the enhancement of key perception. Psychomusicology, 12, 73-83.

Huron, D. (1994). UNIX tools for musical research: the humdrum toolkit reference manual. Stanford, CA: Center for Computer Assisted Research in the Humanities.

Huron, D., \& Parncutt, R. (1993). An improved model of tonality perception incorporating pitch salience and echoic memory. Psychomusicology, 12, 154-171.

Järvinen, T. (1995). Tonal hierarchies in jazz improvisation. Music Perception, 12, 415-437.

Järvinen, T. (1997). Tonal dynamics and metrical structures in jazz improvisation. Published Ph.D. dissertation. Jyväskylä studies in the arts, University of Jyväskylä, Jyväskylä, Finland.

Jernsletten, N. (1978). Om joik og kommunikasjon (Of yoik and communication). By og bygd (Village and countryside). Oslo: Norsk Folkemuseums Årbok.

Jones, M. R. (1981). Music as a stimulus for psychological motion: part 1. Some determinants of expectancies. Psychomusicology, 1, 34-51.

Jones, M. R. (1982). Music as a stimulus for psychological motion: part 2. An expectancy model. Psychomusicology, 2, 1-13.

Jones, M. R. (1990). Learning and the development of expectancies: an interactionist approach. Psychomusicology, 9, 193-228.

Jones, M. R., \& Boltz, M. (1989). Dynamic attention and responses in time. Psychological Review, 96, 459-491.

Jordan, M. I. (1986). Attractor dynamics and parallelism in a connectionist sequential machine. Proceedings of the Eighth Annual Conference of the Cognitive Science Society. Hillsdale, NJ: Erlbaum.

Jouste, M. (1998). Change and metrics in Guhtura-Niillas' luohti. Etnomusikologian vuosikirja (The yearbook of ethnomusicology), 10, 44-63 Special issue: Conference Proceedings of the European Seminar in Ethnomusicology, 1997.

Kantola, T. (1984). Talvadaksen joikuperinne (The yoik tradition of Talvadas). Folkloristiikan tutkimuksia (Studies in folklore). Turku: University of Turku. 
Kessler, E. J., Hansen, C., \& Shepard, R. N. (1984). Tonal schemata in the perception of music in Bali and the West. Music Perception, 2, 131-165.

Kjellström, R., Ternhag, G., \& Rydving, H. (1988). Om jojk (On yoik). Hedemora: Gidlunds Bokförlag.

Knopoff, L., \& Hutchinson, W. (1983). Entropy as a measure of style: the influence of sample length. Journal of Music Theory, 27, 75-97.

Kohonen, T. (1997). Self-organizing maps (2nd ed.). Berlin: Springer.

Krumhansl, C. L. (1990). Cognitive foundations of musical pitch. New York: Oxford University Press.

Krumhansl, C. L. (1991). Melodic structure: theoretical and empirical descriptions. In J. Sundberg, Music, language, speech and brain. London: MacMillian.

Krumhansl, C. L. (1995a). Effects of musical context on similarity and expectancy. Systematische Musikwissenschaft (Systematic musicology), 3, 211-250.

Krumhansl, C. L. (1995b). Music psychology and music theory: problems and prospects. Music Theory Spectrum, 17, 53-90.

Krumhansl, C. L. (1997). Effects of perceptual organization and musical form on melodic expectancies. In M. Leman, Music, gestalt, and computing: studies in cognitive and systematic musicology. Berlin: Springer.

Krumhansl, C. L., \& Kessler, E. J. (1982). Tracing the dynamic changes in perceived tonal organization in a spatial representation of musical keys. Psychological Review, 89, 334-368.

Krumhansl, L. K., Louhivuori, J., Toiviainen, P., Järvinen, T., \& Eerola, T. (1999). Melodic expectancy in Finnish folk hymns: convergence of behavioral, statistical, and computational approaches. Music Perception, 17, 151-197.

Launis, A. (1908). Lappische juoigos-melodien (Lappish yoik melodies). Helsinki: Finno-Ugric Society.

Leman, M. (1995). Music and schema theory: cognitive foundations of systematic musicology. Berlin: Springer.

Lomax, A. (1968). Folk song style and culture. Washington, DC: American Association for the Advancement of Science.

Louhivuori, J., Kaipainen, M., \& Toiviainen, P. (1997). Music, memory, and time - the basis of the universality of musical mind. Finnish Journal of Music Education, 2 (3-4), 15-31.

Lüderwaldt, A. (1976). Joiken aus Norwegen (Yoiks from Norway). Studien zur Charakteristik und gesellschaftlichen Bedeutung des lappischen Gesanges. (Studies of the characteristics and social significance of Lapp song), vol. 2. Bremen: Selbstverlag des Museums.

Meyer, L. B. (1956). Emotion and meaning in music. Chicago, IL: University of Chicago Press.

Narmour, E. (1990). The analysis and cognition of basic melodic structures: the implication-realization model. Chicago, IL: University of Chicago Press.

Oram, N., \& Cuddy, L. L. (1995). Responsiveness of Western adults to pitch-distributional information in melodic sequences. Psychological Research, 57, 103-118.

Saastamoinen, I. (1987). Kolttasaamelainen le'udd (Scolt Sami yoiks). Finnish Yearbook of Ethnomusicology, 1987, 66-95.

Saastamoinen, I. (1994). Rytmikaava-ajattelu saamelaismusiikin hahmotusmallina (The concept of rhythmic patterns as a model for hearing Sami yoiks). Unpublished master's thesis, Department of Music, University of Jyväskylä, Jyväskylä, Finland.

Saastamoinen, I. (1998). Laulu, рии, ruтрu (Song, tree, drum). Unpublished licenciate thesis, Department of Music, University of Jyväskylä, Jyväskylä, Finland.

Schellenberg, E. G. (1996). Expectancy in melody: tests of the implication-realization model. Cognition, $58,75-125$.

Schellenberg, E. G. (1997). Simplifying the implication-realization model of melodic expectancy. Music Perception, 14, 295-318.

Schmuckler, M. A. (1989). Expectation in music: investigation of melodic and harmonic processes. Music Perception, 7, 109-150.

Schmuckler, M. A. (1990). The performance of global expectations. Psychomusicology, 9, 122-147.

Sloboda, J. A. (1991). Musical structure and emotional response: some empirical findings. Psychology of Music, 19, 110-120.

Stockmann, D. (1986). Arbeitsbericht über das Forschungsprojekt 'Musik der Samen' (Report on the 
research project 'Music of the Sami'). Arsskrift 1985 (pp. 50-59). Stockholm: Royal Swedish Academy of Music.

Stockmann, D. (1987). Bericht über eine Informations- und Sammelreise zu den Samen (Report on an information and field work study of the Sami). Arsskrift 1986 (pp. 39-46). Stockholm: Royal Swedish Academy of Music.

Stockmann, D. (1990). Formprinzipen Samischr Joiken und ihre Zusammenhänge mit der Gattungsfunktion (Principles of form in Sami yoiks and their relation to genre function). Probleme der Volksmusikforschung, vol. 5 (pp. 22-46). Bern: Lang.

Thompson, W. F., \& Stainton, M. (1998). Expectancy in Bohemian folk song melodies: evaluation of implicative principles for implicative and closural intervals. Music Perception, 15, 231-252.

Thompson, W. F., Cuddy, L. L., \& Plaus, C. (1997). Expectancies generated by melodic intervals: evaluation of principles of melodic implication in a melody-completion task. Perception \& Psychophysics, 59, 1069-1076.

Tirén, K. (1942). Die lappische Volksmusik (The Lappish folk music). Acta Lapponica, vol. 3. Stockholm: Hugo Gebers.

Treisman, A. M. (1964). Verbal cues, language, and meaning in selective attention. American Journal of Psychology, 77, 206-219.

Unyk, A. M., \& Carlsen, J. C. (1987). The influence of expectancy on melodic perception. Psychomusicology, 7, 3-23.

Valkeapää, N. -A. (1984). Ett sätt att lugna renar (A way of calming reindeers). Tidskrift för Nordisk Litteratur, 24, 43-47. 